QUARTERLY OF APPLIED MATHEMATICS

VOLUME LXIII, NUMBER 1

MARCH 2005, PAGES 117-155

S $0033-569 X(05) 00958-2$

Article electronically published on January 19, 2005

\title{
A PROPOSAL CONCERNING THE PHYSICAL RATE OF DISSIPATION IN MATERIALS WITH MEMORY
}

\author{
BY \\ J. M. GOLDEN
}

School of Mathematical Sciences, Dublin Institute of Technology, Kevin Street, Dublin 8, Ireland

\begin{abstract}
It has been known for several decades that the free energy and entropy of a material with memory is not in general uniquely determined, nor are the total dissipation in the material over a given time period and the rate of dissipation. The dissipation in a material element would in particular seem to be a quantity that has immediate physical objectivity. It must be seen therefore as a significant weakness in the thermodynamics of materials exhibiting memory effects, that a quantity as basic as the rate of dissipation cannot be predicted in terms of the constitutive parameters.

The objective of the present work is to propose a formula for the physical free energy of a linear scalar viscoelastic material in terms of a family of free energies, each of which can be regarded as an estimate of the physical quantity. This formula follows from a new physical hypothesis of Maximum Parametric Symmetries, which states that the physical free energy and dissipation have the closest possible level of symmetry among the parameters of the theory to that of the work function. This results in the assignment of explicit weights to all members of the family of free energies, each of these being associated with a particular factorization of a quantity closely related to the loss modulus of the material. It is interesting that the final formula proposed for the physical free energy can be expressed in simple, closed form. Once the free energy is known, the corresponding physical rate of dissipation can also be determined without difficulty.

It is shown that non-trivial equivalence classes of states, in the sense of Noll, exist only if the material has a relaxation function derivative, the Fourier transform of which has only isolated singularities in the complex frequency plane. The members of the family of free energies used to determine the physical free energy are all functions of such an equivalence class. The derivation of their form is a generalization of work reported in Maximum and minimum free energies for a linear viscoelastic material, Quart. Appl. Math. 60, 341 - 381 (2002).
\end{abstract}

Received June 18, 2004.

2000 Mathematics Subject Classification. Primary 74A15, 74D05; Secondary 30E20.

My thanks to M. Fabrizio, L. Deseri and G. Gentili (deceased December 2000) for several useful conversations.

E-mail address: murrough.golden@dit.ie

(C)2005 Brown University 
1. Introduction. It has been known for several decades that the free energy and entropy of a material with memory is not in general uniquely determined by the constitutive parameters (for example [1]-[18]). A consequence of this is that the total dissipation in the material over a given time period and the rate of dissipation are not uniquely determined either.

Thus, we may have different estimates of the total dissipation over a time interval or, alternatively, the rate of dissipation at a given time, in a material with specified constitutive relations, undergoing a prescribed deformation history (in the isothermal case, for example). Now, in contrast perhaps to free energy or entropy, the rate of dissipation in a material element would seem to be a quantity that has immediate physical objectivity. In particular, for isothermal conditions, the rate of dissipation, due to a specified mechanical process, is simply the rate of heat production in the element, by that process.

It must be seen therefore as a significant weakness in the thermodynamics of materials exhibiting memory effects that a quantity as basic as the rate of dissipation cannot be predicted in terms of the constitutive parameters.

The objective of the present work is to propose a formula for the physical free energy of a linear viscoelastic material in terms of various estimates of this quantity. This formula follows from a new physical hypothesis, which results in the assignment of explicit weights to each member of a family of free energies, each of these being associated with a particular factorization of the function $H(\omega)$ used in earlier work [14, 13, 17, 20, 19, 18]. Once the free energy is known, the rate of dissipation can also be determined without difficulty. It is interesting that the final formulae can be expressed in simple closed form, rather than as a summation over the estimating quantities.

The main analysis takes place under the assumption that the relaxation function consists of a sum of terms, each of which is a polynomial multiplying a strictly decaying exponential and trigonometric functions, or, in the frequency domain, the case where $G_{F}^{\prime}$, the Fourier transform of the derivative of the relaxation function, contains only isolated singularities on the upper half of the complex frequency plane; it must always be analytic on the lower half-plane. (This generalizes what has been referred to as the discrete spectrum case in earlier work, namely where the relaxation function $G^{\prime}$ is a sum of strictly decaying exponentiald $]$ or $G_{F}^{\prime}$ is a sum of simple poles on the positive imaginary axis.) It is pointed out that an arbitrary relaxation function can be well approximated by such functions; and the choosing of a form for a relaxation function is normally a phenomenological exercise, with perhaps some guidance from molecular models.

The other main possibility is a generalization of what has been referred to as the continuous spectrum case [21], namely an integral over strictly decaying exponentials, which corresponds to $G_{F}^{\prime}$ containing branch cuts on the positive imaginary axis. The generalization allows these branch cuts to be off the imaginary axis. It is shown that singularities of this sort imply that the maximum free energy must be the work function, with associated rate of dissipation equal to zero.

\footnotetext{
${ }^{1}$ We shall adopt the terminology that strictly decaying exponentials means expressions such as $e^{-\alpha t}$ where $t>0$ and $\alpha$ is real and that decaying exponentials refers to where Re $\alpha>0$ and Im $\alpha$ need not be zero.
} 
This is an unattractive feature in that the zero rate of dissipation is clearly unphysical. Also, the total dissipation associated with the maximum free energy provides a lower bound on the total physical dissipation. When the maximum free energy is the work function, this lower bound is zero, which is the weakest possible estimate. Hence, it is argued that excluding branch cuts and allowing only isolated singularities on the finite complex plane is more desirable, from a theoretical point of view. For such materials, it is shown that the maximum free energy is less than the work function and is a function of state in the sense defined by [22, 23, 12, 24, 17], or in terms of a concept discussed in Section 2 a function of the minimal state.

This restriction is no less desirable from a practical viewpoint, in that it amounts to approximating $G_{F}^{\prime}$ as a rational function of frequency. A given set of experimental data can generally be well approximated by such a function.

It must be pointed out however that continuous spectrum, rather than discrete spectrum models have typically been used in experimental work [25].

For decades, researchers, faced with the need to estimate dissipation, have commonly used a quadratic functional of strain histories with a kernel of the form $G^{\prime \prime}\left(t_{1}+t_{2}\right)$ [26] which is motivated by the work of Staverman and Schwarzl 1, based on mechanical models (see also [27, 28, 29]). It is shown in [1] that this functional is a free energy only if the relaxation function is monotonic.

The formula presented here is a free energy under considerably wider conditions on the relaxation function (see Section 2). The motivation for it is based on a new physical assumption, the hypothesis of Maximum Parametric Symmetries, which states that the physical free energy and dissipation in the theory have the highest level of symmetry, among the parameters of the theory, achievable with a certain formula involving sums of approximations to this free energy. Given the required symmetries, we deduce with the aid of certain weak and natural assumptions, an explicit formula for this quantity. Thus, this new approach is quite different from and more abstract than that leading to the Staverman-Schwartzl free energy but does have wider validity.

On the question: how does one finally decide on the validity of the formula presented, the answer would seem to be by experiment. Whether this is practical or not is unclear to the present author.

In order to focus on the essential developments and to keep algebraic complexity to a minimum, the discussion is confined to the case of scalar constitutive relations. In fact, however, the generalization to tensor constitutive relations is relatively straightforward, at least under the assumption of time-independent eigenspaces for relaxation functions 13. For each eigenspace, the scalar formulae apply.

The arguments presented can all be generalized, in a very straightforward manner, to the case where the constitutive equations are non-linear in elastic effects with linear memory terms.

The isothermal case is considered in this paper. The generalization to a non-isothermal context will be considered in a later paper, incorporating tensor constitutive relations with non-linear equilibrium terms. 
In Section 2, constitutive equations, minimal viscoelastic states and other fundamental relationships and concepts are presented. Properties of free energies are discussed and an expression for the minimum free energy is given.

In Section 3, it is pointed out that the relaxation function is determined by the singularity structure in the complex frequency domain of its Fourier transform. In particular, the case of isolated singularities is discussed in detail. In Section 4, a number of results are obtained, mainly for materials characterized only by isolated singularities, while an expression is obtained for the maximum free energy in Section 5 .

In Section 6 free energies which are functions of the minimal state are expressed as finite quadratic forms while in Section 7 a family of free energies consisting of minimum, maximum and intermediate free energies is presented. Explicit forms for these quantities are derived in Section 8

In Section 9 expressions for the proposed physical free energy and rate of dissipation are derived.

Apart from Section 9 this work is largely a generalization of Part B of [17] (see also 20]) from the case of simple poles to general isolated singularities, though the derivation of the maximum free energy in Section 5 is simpler. The summary of the abstract form of thermodynamics given in [17] provides general definitions of the minimum and maximum free energies, which are important in the present work.

2. Basic relationships and free energies. We consider a linear viscoelastic solid, subject to stress in such a way that there is only one non-zero component of stress $T(t)$ and strain $E(t)$ related by

$$
\begin{aligned}
T(t) & =G_{0} E(t)+\int_{0}^{\infty} G^{\prime}(s) E^{t}(s) d s, \quad E^{t}(s)=E(t-s), \quad s \in \mathbb{R}, \\
& =G_{\infty} E(t)+\int_{0}^{\infty} G^{\prime}(s) E_{r}^{t}(s) d s, \quad E_{r}^{t}(s)=E^{t}(s)-E(t)
\end{aligned}
$$

where $E^{t} \in L^{1}\left(\mathbb{R}^{+}\right) \cap L^{2}\left(\mathbb{R}^{+}\right) \cap C^{1}\left(\mathbb{R}^{+}\right)$and $G^{\prime} \in L^{1}\left(\mathbb{R}^{+}\right) \cap L^{2}\left(\mathbb{R}^{+}\right)$using the notation here and below: $\mathbb{R}$ is the set of reals, $\mathbb{R}^{+}$the positive reals, and $\mathbb{R}^{++}$the strictly positive reals; similarly $\mathbb{R}^{-}, \mathbb{R}^{--}$are the negative and strictly negative reals. The relaxation function

$$
G(s)=G_{0}+\int_{0}^{s} G^{\prime}(u) d u
$$

is then well defined along with $G_{\infty}=\lim _{s \rightarrow \infty} G(s)$. We take

$$
G_{\infty}>0
$$

so that the body is a solid.

We shall use $(2.1)_{2}$ for later developments. Relation $(2.1)_{1}$ was the basis of the early papers on this topic 14, 13, 17]. However, in more recent work, for example [18, 30, 19] 31, formulae based on the relative history have been used because they yield explicitly positive free energies. Also, various quantities have better convergence properties in the frequency domain. 
Let $\Omega$ be the complex $\omega$ plane and let

$$
\begin{aligned}
\Omega^{+} & =\left\{\omega \in \Omega \mid \operatorname{Im}(\omega) \in \mathbb{R}^{+}\right\}, \\
\Omega^{(+)} & =\left\{\omega \in \Omega \mid \operatorname{Im}(\omega) \in \mathbb{R}^{++}\right\} .
\end{aligned}
$$

These define the upper half-plane including and excluding the real axis, respectively. Similarly, $\Omega^{-}, \Omega^{(-)}$are the lower half-planes including and excluding the real axis, respectively.

A viscoelastic state is defined in general by the current value of strain and the history $\left(E(t), E^{t}\right)$. The concept of a minimal state, defined in [17] (see also [22, 23, 24, 12, 11, 13]) can be expressed as follows: two viscoelastic states $\left(E_{1}(t), E_{1}^{t}\right),\left(E_{2}(t), E_{2}^{t}\right)$ are equivalent or in the same equivalence class or minimal state if

$$
E_{1}(t)=E_{2}(t) ; \quad \int_{0}^{\infty} G^{\prime}(s+\tau)\left[E_{1}^{t}(s)-E_{2}^{t}(s)\right] d s=0 \quad \forall \tau \geq 0 .
$$

A functional of $\left(E(t), E^{t}\right)$ which yields the same value for all members of the same minimal state will be referred to as a function of the minimal state or as a minimal state variable. We can replace the histories $E_{1}^{t}, E_{2}^{t}, E^{t}$ in these statements by the relative histories $E_{1 r}^{t}, E_{2 r}^{t}, E_{r}^{t}$.

For any $f \in L^{2}(\mathbb{R})$, we denote its Fourier transform by

$$
f_{F}(\omega)=\int_{-\infty}^{\infty} f(\xi) e^{-i \omega \xi} d \xi, f_{F} \in L^{2}(\mathbb{R}) .
$$

If $f$ is a real-valued function in the time domain - which will be the case for all functions of interest here - then

$$
\bar{f}_{F}(\omega)=f_{F}(-\omega)
$$

where the bar denotes complex conjugate. We have

$$
\begin{aligned}
& f_{F}(\omega)=f_{+}(\omega)+f_{-}(\omega), \\
& f_{+}(\omega)=\int_{0}^{\infty} f(\xi) e^{-i \omega \xi} d \xi, \\
& f_{-}(\omega)=\int_{-\infty}^{0} f(\xi) e^{-i \omega \xi} d \xi, \quad f_{ \pm} \in L^{2}(\mathbb{R}),
\end{aligned}
$$

where $f_{+}$is analytic in $\Omega^{(-)}$. For relevant functions in the present work, we also assume that it is analytic on $\mathbb{R}$ and thus on $\Omega^{-}$or, strictly, an open set containing $\Omega^{-}$. Similarly, $f_{-}$is analytic on an open set containing $\Omega^{+}$.

Functions on $\mathbb{R}$ which vanish identically on $\mathbb{R}^{--}$are defined as functions on $\mathbb{R}^{+}$. For such quantities, $f_{F}=f_{c}-i f_{s}$ where $f_{c}, f_{s}$ are the Fourier cosine and sine transforms

$$
\begin{aligned}
& f_{c}(\omega)=\int_{0}^{\infty} f(\xi) \cos \omega \xi d \xi=f_{c}(-\omega), \\
& f_{s}(\omega)=\int_{0}^{\infty} f(\xi) \sin \omega \xi d \xi=-f_{s}(-\omega) .
\end{aligned}
$$

Thus

$$
G_{F}^{\prime}(\omega)=\int_{0}^{\infty} G^{\prime}(s) e^{-i \omega s} d s=G_{c}^{\prime}(\omega)-i G_{s}^{\prime}(\omega)
$$


For the developments in this work, we shall assume that $G_{F}^{\prime}$ is analytic at infinity. In the case where $G^{\prime}$ has finite memory components [19], referred to briefly in Section 4 this is not true. The property of $G_{F}^{\prime}$ that

$$
\lim _{\omega \rightarrow \infty} i \omega G_{F}^{\prime}(\omega)=G^{\prime}(0)
$$

will be required. Properties of $G_{s}^{\prime}(\omega)$ include

$$
\begin{aligned}
G_{s}^{\prime}(\omega) & \leq 0 \quad \forall \omega \in \mathbb{R}^{++}, \\
G_{s}^{\prime}(-\omega) & =-G_{s}^{\prime}(\omega), \quad \forall \omega \in \mathbb{R},
\end{aligned}
$$

the first relation being a consequence of the second law of thermodynamics [9] and the second being a particular case of $(2.8)_{2}$. It follows that $G_{s}^{\prime}(0)=0$. We also have [10]

$$
G_{\infty}-G_{0}=\frac{1}{\pi} \int_{-\infty}^{\infty} \frac{G_{s}^{\prime}(\omega)}{\omega} d \omega<0
$$

so that $G_{s}^{\prime}(\omega) / \omega \in L^{1}(\mathbb{R})$. It follows from (2.2) and (2.12) that $G_{0}$ is positive.

The function $G_{F}^{\prime}(\omega)$ is analytic on $\Omega^{(-)}$. This is a consequence of the fact that $G^{\prime}$ vanishes on $\mathbb{R}^{--}$which is essentially the requirement of causality [29].

Referring to the assumption after (2.7), we have that $G_{F}^{\prime}(\omega)$ is analytic on $\mathbb{R}$ and therefore on $\Omega^{-}$. This implies that any singularities are at least slightly removed into $\Omega^{(+)}$, which in turn means that $G^{\prime}$ decays exponentially at large positive times. However, formulae of physical interest will generally be continuous with respect to taking the limit to non-exponential behaviour.

For $\omega \in \Omega^{(+)}$, relation $(2.9) 1$ cannot in general be used to define $G_{F}^{\prime}(\omega)$. Instead, it is defined by analytic continuation from the region of analyticity, namely $\Omega^{-}$and possibly a strip of $\Omega^{(+)}$.

Because $G^{\prime}$ is real, we have from (2.9)

$$
\overline{G_{F}^{\prime}(\omega)}=G_{F}^{\prime}(-\bar{\omega}) .
$$

This constraint in fact means that the singularities are symmetric under reflection in the positive imaginary axis.

The quantity $\bar{G}_{F}^{\prime}(\omega)$ is analytic in $\Omega^{+}$, its singularity structure being a reflection in the origin (and indeed, by virtue of the observation of the previous paragraph, a mirror image, in the real axis) of that of $G_{F}^{\prime}(\omega)$. Thus, $G_{s}^{\prime}(\omega)$ has singularities in both $\Omega^{(+)}$and $\Omega^{(-)}$which are mirror images of one another. Similarly, its zeros will be mirror images of one another. We will be interested in the singularity structure of

$$
\begin{aligned}
H(\omega) & =\frac{\omega}{2 i}\left(G_{F}^{\prime}(\omega)-\bar{G}_{F}^{\prime}(\omega)\right)=-\omega G_{s}^{\prime}(\omega) \\
& =H(-\omega) \geq 0 \quad \forall \omega \in \mathbb{R} .
\end{aligned}
$$

The non-negativity of $H$ follows from (2.11). We have $H(\omega)=H_{1}\left(\omega^{2}\right)$ so that $H(\omega)$ goes to zero at least quadratically at the origin. It is assumed that the behaviour is in fact quadratic, i.e. $H(\omega) / \omega^{2}$ tends to a finite, non-zero quantity as $\omega$ tends to zero. The singularities of $H$ are the same as those of $G_{F}^{\prime}$ in $\Omega^{(+)}$and of $\bar{G}_{F}^{\prime}$ in $\Omega^{(-)}$. It will be required in later developments that $H(\omega)$ can be written in the form

$$
H(\omega)=H_{+}(\omega) H_{-}(\omega)
$$


where $H_{+}(\omega)$ has no singularities or zeros in $\Omega^{(-)}$and is thus analytic in $\Omega^{-}$. Similarly, $H_{-}(\omega)$ is analytic in $\Omega^{+}$with no zeros in $\Omega^{(+)}$. Thus the singularities of $G_{F}^{\prime}$ must all occur in $H_{+}$and those of $\bar{G}_{F}^{\prime}$ in $H_{-}$. There may be other singularities in $H_{ \pm}$which cancel on multiplication. That such a factorization is always possible is shown for general tensor constitutive relations in 13 .

Using (2.10) and (2.14), one can show that

$$
H_{\infty}=\lim _{|\omega| \rightarrow \infty} H(\omega)=-G^{\prime}(0) \geq 0 .
$$

The sign of $G^{\prime}(0)$ has been deduced by various authors from thermodynamic constraints in the general three-dimensional case [32, 33, 9]. We assume for present purposes that $G^{\prime}(0)$ is non-zero so that $H_{\infty}$ is a finite, positive number. Then $H(\omega) \in \mathbb{R}^{++} \forall \omega \in$ $\mathbb{R}, \omega \neq 0$.

The factorization (2.15) is unique up to a constant phase factor. We put [14]

$$
\begin{aligned}
H_{ \pm}(\omega) & =H_{\mp}(-\omega)=\bar{H}_{\mp}(\omega), \\
H(\omega) & =\left|H_{ \pm}(\omega)\right|^{2},
\end{aligned}
$$

one consequence of which is that the factorization is now unique up to a change of sign.

A general method is outlined in [14 for determining the factors of $H$, when $G_{F}^{\prime}$ is analytic at infinity.

Consider now the strain history $E^{t}$. Define

$$
E_{+}^{t}(\omega)=\int_{0}^{\infty} E^{t}(s) e^{-i \omega s} d s, \quad E_{+}^{t} \in L^{2}(\mathbb{R}) .
$$

It is analytic in $\Omega^{(-)}$, a property which will be extended to $\Omega^{-}$in accordance with the observation after (2.7). It is defined on $\Omega^{(+)}$, excluding singular points, by analytic continuation. We also require the Fourier transform of the relative history:

$$
\begin{aligned}
E_{r+}^{t}(\omega) & =E_{+}^{t}(\omega)-E(t) \int_{0}^{\infty} e^{-i \omega s} d s \\
& =E_{+}^{t}(\omega)-\frac{E(t)}{i \omega^{-}} ; \quad \omega^{-}=\lim _{\alpha \rightarrow 0^{+}}(\omega-i \alpha)
\end{aligned}
$$

where the limit being taken after any integration involving the quantity $\left(\omega^{-}\right)^{-1}$ has been carried out. Under a similar assumption to that for $E_{+}^{t}$, it may be concluded that $E_{r+}^{t}$ is analytic in $\Omega^{-}$.

We also consider the continuation $E^{t}$ on $\mathbb{R}^{-}$, which, for our purposes, will be a derived quantity with the property that $E^{t}(-\infty)$ is not zero. However, defining

$$
E_{1}^{t}(s)=E^{t}(s)-E_{\infty}, \quad s \in R^{-}, \quad E_{\infty}=E^{t}(-\infty),
$$

we have that $E_{1}^{t} \in L^{1}\left(\mathbb{R}^{-}\right) \cap L^{2}\left(\mathbb{R}^{-}\right) \cap C^{1}\left(\mathbb{R}^{-}\right)$. Let

$$
\begin{aligned}
E_{-}^{t}(\omega) & =E_{1-}^{t}(\omega)+E_{\infty} \int_{-\infty}^{0} e^{-i \omega s} d s \\
& =E_{1-}^{t}(\omega)-\frac{E_{\infty}}{i \omega^{+}}, \quad \omega^{+}=\lim _{\alpha \rightarrow 0^{+}}(\omega+i \alpha), \\
E_{1-}^{t}(\omega) & =\int_{-\infty}^{0} d s E_{1}^{t}(s) e^{-i \omega s}
\end{aligned}
$$


where the limit is to be taken after any integration involving the quantity $\left(\omega^{+}\right)^{-1}$. Under a similar assumption to that for $E_{+}^{t}$, we may conclude that $E_{1-}^{t}$ is analytic in $\Omega^{+}$. It therefore follows that $E_{-}^{t}(\omega)$ is analytic in $\Omega^{+}$.

We write

$$
\begin{aligned}
E_{F}^{t}(\omega) & =E_{+}^{t}(\omega)+E_{-}^{t}(\omega) \\
& =\int_{-\infty}^{\infty} d s E^{t}(s) e^{-i \omega s} .
\end{aligned}
$$

Analyticity at infinity is assumed for $E_{ \pm}^{t}(\omega)$. We also define

$$
E_{r-}^{t}(\omega)=E_{-}^{t}(\omega)+\frac{E(t)}{i \omega^{+}} .
$$

The quantity $E_{r-}^{t}$ is the Fourier transform of $E^{t}(s)-E(t), s \leq 0$.

Derived histories (see Section 7) which do not vanish in the distant past obey formulae which are the complex conjugates of (2.19).

Note that

$$
\frac{d E_{+}^{t}(\omega)}{d t}=-i \omega E_{+}^{t}(\omega)+E(t)
$$

which gives

$$
\frac{d E_{r+}^{t}(\omega)}{d t}=-i \omega E_{r+}^{t}(\omega)-\frac{\dot{E}(t)}{i \omega^{-}} .
$$

Applying Plancherel's theorem to (2.1) 2 , we obtain

$$
T(t)=G_{\infty} E(t)+\frac{1}{2 \pi} \int_{-\infty}^{\infty} \bar{G}_{F}^{\prime}(\omega) E_{r+}^{t}(\omega) d \omega .
$$

Observe that if we replace $\bar{G}_{F}^{\prime}(\omega)$ by $\left[\bar{G}_{F}^{\prime}(\omega)+F(\omega)\right]$ where $F(\omega)$ is analytic on $\Omega^{-}$and goes to zero at large frequencies as rapidly as $\omega^{-1}$, the relationship still holds. This follows by a simple application of Cauchy's theorem. In particular, we have

$$
T(t)=G_{\infty} E(t)+\frac{1}{2 \pi} \int_{-\infty}^{\infty}\left[\bar{G}_{F}^{\prime}(\omega)+\lambda G_{F}^{\prime}(\omega)\right] E_{r+}^{t}(\omega) d \omega,
$$

where $\lambda$ is any complex constant. Choosing $\lambda=-1$ yields

$$
T(t)=G_{\infty} E(t)+\frac{i}{\pi} \int_{-\infty}^{\infty} G_{s}^{\prime}(\omega) E_{r+}^{t}(\omega) d \omega .
$$

In fact, 2.23) corresponds to taking the even extension of $G$ to $\mathbb{R}$, namely $G(s)=$ $G(|s|), s \in \mathbb{R}$, which yields the odd extension of $G^{\prime}$. This amounts to writing $(2.1)_{2}$ as

$$
T(t)=G_{\infty} E(t)+\int_{-\infty}^{\infty} \frac{d}{d s} G(|s|) E_{r}^{t}(s) d s
$$

noting that $E_{r}^{t}$ is taken to be zero on $\mathbb{R}^{-}$. Then we can derive (2.23) by observing that

$$
\int_{-\infty}^{\infty} \frac{d}{d s} G(|s|) e^{-i \omega s} d s=-2 i G_{s}^{\prime}(\omega) .
$$

The work done on the material by the strain history $E^{t}$ is

$$
\widetilde{W}\left(E(t), E^{t}\right)=\int_{-\infty}^{t} T(\tau) \dot{E}(\tau) d \tau
$$


It will be clear from the representation of $\widetilde{W}\left(E(t), E^{t}\right)$ in the frequency domain, given below, that it is a non-negative quantity. We will restrict our considerations to histories such that $\widetilde{W}\left(E(t), E^{t}\right)<\infty$. The quantity $\widetilde{W}\left(E(t), E^{t}\right)$ is, in some circumstances, the maximum free energy $([12,11]$ and see Section 44). It will be denoted by $W(t)$. One can show that

$$
\begin{gathered}
W(t)=\phi(t)+\frac{1}{2} \int_{0}^{\infty} \int_{0}^{\infty} E_{r}^{t}\left(s_{1}\right) G_{12}\left(\left|s_{1}-s_{2}\right|\right) E_{r}^{t}\left(s_{2}\right) d s_{1} d s_{2}, \\
G_{12}\left(\left|s_{1}-s_{2}\right|\right)=\frac{\partial}{\partial s_{1}} \frac{\partial}{\partial s_{2}} G\left(\left|s_{1}-s_{2}\right|\right), \\
\phi(t)=\tilde{\phi}(E(t))=\frac{1}{2} G_{\infty} E^{2}(t) .
\end{gathered}
$$

From (2.24) and Plancherel's theorem, we have

$$
W(t)=\phi(t)+\frac{1}{2 \pi} \int_{-\infty}^{\infty} H(\omega)\left|E_{r+}^{t}(\omega)\right|^{2} d \omega
$$

which is a non-negative quantity by virtue of (2.14).

Certain properties of a free energy were derived in [34]. These have been used to characterize a free energy in 9, 14, 13, for example. They have been referred to as the Graffi [35] definition of (or conditions for) a free energy and are given as follows. Let $\psi(t)=\tilde{\psi}\left(E(t), E_{r}^{t}\right)$ be a free energy. Then

P1:

$$
\frac{\partial \psi(t)}{\partial E(t)}=T(t)
$$

P2: Consider a static history equal to $E_{0}$ at all past times. Then

$$
\tilde{\psi}\left(E_{0}, 0\right)=\tilde{\phi}\left(E_{0}\right) \text {. }
$$

where $\tilde{\phi}\left(E_{0}\right)$ is the elastic free energy defined by $(2.26)_{3}$.

P3: For any relative history $E_{r}^{t}$

$$
\tilde{\psi}\left(E(t), E_{r}^{t}\right) \geq \tilde{\phi}(E(t)) .
$$

P4: It is assumed that $\psi$ is differentiable. For any $\left(E(t), E_{r}^{t}\right)$

$$
\dot{\psi}(t)=T(t) \dot{E}(t)-D(t)
$$

where $D(t) \geq 0$. This is an expression of the second law.

The Graffi definition and the general definition of Coleman and Owen [4, 5] are compared in the context of a linear theory in [11].

Let

$$
\begin{aligned}
P^{t}(\omega) & =H_{-}(\omega) E_{r+}^{t}(\omega) \\
& =p_{-}^{t}(\omega)-p_{+}^{t}(\omega)
\end{aligned}
$$

where

$$
p^{t}(z)=\frac{1}{2 \pi i} \int_{-\infty}^{\infty} d \omega \frac{P^{t}(\omega)}{\omega-z}
$$

and $p_{-}^{t}(\omega)$ is the limit of $p^{t}(z)$ on the real axis from above. It is analytic in $\Omega^{(+)}$. Also, $p_{+}^{t}(\omega)$ is the limit from below and is analytic in $\Omega^{(-)}$. Both are analytic on $\mathbb{R}$ by virtue of an argument given in [14], based on the assumed analyticity of $H_{-}$and $E_{r+}^{t}$ on $\mathbb{R}$. 
Relations (2.32) and (2.33) follow from the Plemelj formulae [36]. From (2.27) and (2.32) we have

$$
\begin{aligned}
W(t) & =\phi(t)+\frac{1}{2 \pi} \int_{-\infty}^{\infty} d \omega\left|p_{+}^{t}(\omega)-p_{-}^{t}(\omega)\right|^{2} \\
& =\phi(t)+\frac{1}{2 \pi} \int_{-\infty}^{\infty} d \omega\left[\left|p_{+}^{t}(\omega)\right|^{2}+\left|p_{-}^{t}(\omega)\right|^{2}\right],
\end{aligned}
$$

the second relation following from the analyticity properties of $p_{ \pm}^{t}[14$.

The choice of $E_{r-}^{t}$ which maximizes the recoverable work is given by [14 13, 17, 20, 19, 18, 31,

$$
\begin{aligned}
E_{m}^{t}(\omega) & =-\frac{p_{-}^{t}(\omega)}{H_{-}(\omega)} \\
& =-\frac{1}{2 \pi i} \frac{1}{H_{-}(\omega)} \int_{-\infty}^{\infty} d \omega^{\prime} \frac{H_{-}\left(\omega^{\prime}\right) E_{r+}^{t}\left(\omega^{\prime}\right)}{\omega^{\prime}-\omega^{+}} .
\end{aligned}
$$

The minimum free energy $\psi_{m}(t)$, which is the maximum recoverable work ([10, 17], for example), is given by [14, 13]

$$
\begin{aligned}
\psi_{m}(t) & =\phi(t)+\frac{1}{2 \pi} \int_{-\infty}^{\infty} H(\omega)\left|E_{m}^{t}(\omega)\right|^{2} d \omega \\
& =\phi(t)+\frac{1}{2 \pi} \int_{-\infty}^{\infty}\left|p_{-}^{t}(\omega)\right|^{2} d \omega \leq W(t) .
\end{aligned}
$$

It can be shown that $p_{-}^{t}$ is a function of the minimal state [13], from which it follows that $\psi_{m}(t)$ is, also. The rate of dissipation is

$$
D_{m}(t)=T(t) \dot{E}(t)-\dot{\psi}_{m}(t)=\left|K_{m}(t)\right|^{2}
$$

where

$$
K_{m}(t)=\frac{1}{2 \pi} \int_{-\infty}^{\infty} d \omega H_{-}(\omega) E_{r+}^{t}(\omega)
$$

The quantity $K_{m}(t)$ is real. Clearly, $D_{m}$ is positive, as required by the second law. Relation (2.37) can be shown using special cases of relations (4.13)-4.15) below.

It is easy to show that $\psi_{m}(t)$ obeys the Graffi conditions. Properties P2 and P3 are immediately apparent. $\mathrm{P} 4$ follows from the fact that $D_{m}$ is non-negative. Property P1 can be proved with the aid of

$$
\frac{\partial p_{-}^{t}(\omega)}{\partial E(t)}=-\frac{H_{-}(\omega)}{i \omega}
$$

and the fact that, using (2.32), the frequency integral in (2.23) can be written as

$$
\begin{aligned}
-\frac{i}{\pi} \int_{-\infty}^{\infty} \frac{H(\omega)}{\omega} E_{r+}^{t}(\omega) d \omega & =-\frac{i}{\pi} \int_{-\infty}^{\infty} \frac{H_{+}(\omega)}{\omega}\left[p_{-}^{t}(\omega)-p_{+}^{t}(\omega)\right] d \omega \\
& =-\frac{i}{\pi} \int_{-\infty}^{\infty} \frac{H_{+}(\omega)}{\omega} p_{-}^{t}(\omega) d \omega,
\end{aligned}
$$

since the term involving $p_{+}^{t}$ vanishes by Cauchy's theorem.

It is shown in [13] that $\psi_{m}$ is a free energy also under the definition of Coleman and Owen [4, 5]. 
We can write $\psi_{m}(t)$ in the form

$$
\begin{gathered}
\psi(t)=\phi(t)+\frac{i}{4 \pi^{2}} \int_{-\infty}^{\infty} \frac{\bar{E}_{r+}^{t}\left(\omega_{1}\right) L_{m}\left(\omega_{1}, \omega_{2}\right) E_{r+}^{t}\left(\omega_{2}\right)}{\omega_{1}^{+}-\omega_{2}^{-}} d \omega_{1} d \omega_{2}, \\
L_{m}\left(\omega_{1}, \omega_{2}\right)=H_{+}\left(\omega_{1}\right) H_{-}\left(\omega_{2}\right),
\end{gathered}
$$

by the same manipulations as were used in 14,30 for the actual rather than the relative histories. The notation in the denominator of the integral in (2.40) means that if we integrate first over $\omega_{1}$, it becomes $\left(\omega_{1}-\omega_{2}^{-}\right)$or if $\omega_{2}$ first, then it is $\left(\omega_{1}^{+}-\omega_{2}\right)$. Also, $D_{m}(t)$, given by (2.37), can be expressed as

$$
D_{m}(t)=\frac{1}{4 \pi^{2}} \int_{-\infty}^{\infty} \bar{E}_{r+}^{t}\left(\omega_{1}\right) L_{m}\left(\omega_{1}, \omega_{2}\right) E_{r+}^{t}\left(\omega_{2}\right) d \omega_{1} d \omega_{2} .
$$

3. The form of the relaxation function derivative. A material can be characterized by the singularity structure of $G_{F}^{\prime}$ on $\Omega^{(+)}$, as may be seen, at least in the case where $G_{F}^{\prime}$ is analytic at infinity, by evaluating

$$
\begin{aligned}
& G^{\prime}(s)=\frac{1}{2 \pi} \int_{-\infty}^{\infty} G_{F}^{\prime}(\omega) e^{i \omega s} d \omega, \quad s \in \mathbb{R}^{++}, \\
& G^{\prime}(s)=0, \quad s \in \mathbb{R}^{--}, \quad G^{\prime}(0)=G^{\prime}\left(0^{+}\right),
\end{aligned}
$$

by contour integration on $\Omega^{+}$.

The possible types of singularities are: isolated singularities, discontinuities associated with branch cuts and essential singularities.

Let us first consider in some detail the case where $G_{F}^{\prime}$ has only isolated singularities. We confine the discussion to the case where the number of such singularities is finite, not only to avoid convergence issues but also in recognition of the fact that the determination of $G_{F}^{\prime}$ for a given material is always, at least partially, an exercise in phenomenology, in effect, curve fitting to data point:2. An approximation to $G_{F}^{\prime}$ by a rational function would be required to model singularities. Clearly, the outcome of such an exercise automatically produces a finite, rather than an infinite number of singularities.

Such a rational function can be expressed as a sum of pole terms of varying orders. These correspond to $G^{\prime}$ consisting of exponential functions multiplying polynomials. One can see this by multiple differentiations of $G_{F}^{\prime}$, made up of a sum of simple poles, with respect to the positions of the poles. This corresponds to the differentiation of $G^{\prime}(s)$ with respect to the coefficients of $s$ in the exponentials. If the poles are simple and on the imaginary axis of $\Omega^{(+)}$, we obtain, before differentiation, sums of strictly decaying exponentials (i.e. decaying exponentials in the sense of footnote 1) in $G^{\prime}$, and after differentiation, strictly decaying exponentials multiplied by polynomials. If the singularities are off the positive imaginary axis, one obtains decaying exponentials multiplying polynomials. The positions of the singularities are subject to the condition that $G^{\prime}$ be real, namely (2.13).

\footnotetext{
${ }^{2}$ Predictions of molecular theories, such as those based on Brownian motion, may also provide guidance [25].
} 
We write

$$
G_{F}^{\prime}(\omega)=\sum_{l=1}^{S} \sum_{j=1}^{m_{l}} \frac{r_{l j}}{\left(\zeta_{l}-\omega\right)^{j}}, \quad \zeta_{l} \in \Omega^{(+)}
$$

corresponding to

$$
\begin{gathered}
G^{\prime}(s)=\sum_{l=1}^{S} g_{l}(s) e^{i \zeta_{l} s}, \quad g_{l}(s)=\sum_{j=0}^{m_{l}-1} c_{j}^{l} s^{J}, \\
c_{j-1}^{l}=\frac{r_{l j}(-i)^{j}}{(j-1) !},
\end{gathered}
$$

where $S$ is the number of points where singularities occur and $m_{l}$ is the highest order singularity at the point $\zeta_{l}$. Individual values of $r_{l j}$ may of course be zero, though not for $j=m_{l}$ which defines the highest power at a given singular point. Some values of $r_{1 j}$ must be non-zero, by virtue of (2.10). Note that the form (3.1) could be deduced by applying the partial fractions theorem to a rational function of $\omega$ with the correct behaviour at infinity, namely that implied by (2.10).

In order to satisfy (2.13), we write (3.1) in the form

$$
\begin{aligned}
G_{F}^{\prime}(\omega) & =\sum_{l=1}^{I} \sum_{j=1}^{m_{l}} \frac{g_{l j}}{\left(\zeta_{l}-\omega\right)^{j}} \\
& +\sum_{l=I+1}^{T} \sum_{j=1}^{m_{l}}\left\{\frac{r_{l j}}{\left(\zeta_{l}-\omega\right)^{j}}+\frac{\bar{r}_{l j}}{\left(\bar{\zeta}_{l}+\omega\right)^{j}}\right\}, \\
\zeta_{l} & =i \alpha_{l}, \alpha_{l} \in \mathbb{R}^{++}, l=1,2, \ldots, I, g_{l j}=(-1)^{j} \bar{g}_{l j}, \\
\zeta_{l} & \in \Omega^{(+)},\left|\operatorname{Re} \zeta_{l}\right|>0, l=I+1, \ldots, T, \quad r_{l j}=(-1)^{j} \bar{r}_{l j} .
\end{aligned}
$$

Thus, there are $I$ singularities on the imaginary axis and $M=T-I$ pairs of singularities at $\left(\zeta_{l},-\bar{\zeta}_{l}\right), l=I+1, \ldots, T$, so that $S=I+2 M$.

We can write $G_{F}^{\prime}$ as a rational function

$$
G_{F}^{\prime}(\omega)=\frac{P(\omega)}{Q(\omega)}
$$

where

$$
\begin{aligned}
Q(\omega) & =\prod_{l=1}^{I}\left(\omega-\zeta_{l}\right)^{m_{l}} \prod_{l=I+1}^{T}\left(\omega-\zeta_{l}\right)^{m_{l}}\left(\omega+\bar{\zeta}_{l}\right)^{m_{l}} \\
& =\prod_{l=1}^{I}\left(\omega-\zeta_{l}\right)^{m_{l}} \prod_{l=I+1}^{T}\left(\omega^{2}-\left|\zeta_{l}\right|^{2}-2 i \omega \operatorname{Im} \zeta_{l}\right)^{m_{l}} .
\end{aligned}
$$

Also, we write $P$ in the form

$$
\begin{aligned}
P(\omega) & =G^{\prime}(0) \prod_{l=1}^{I_{z}}\left(\omega-\xi_{l}\right)^{p_{l}} \prod_{l=I_{z}+1}^{T_{z}}\left(\omega-\xi_{l}\right)^{p_{l}}\left(\omega+\bar{\xi}_{l}\right)^{p_{l}} \\
& =\prod_{l=1}^{I_{z}}\left(\omega-\xi_{l}\right)^{p_{l}} \prod_{l=I_{z}+1}^{T_{z}}\left(\omega^{2}-\left|\xi_{l}\right|^{2}-2 i \omega \operatorname{Im} \xi_{l}\right)^{m_{l}}
\end{aligned}
$$


where

$$
\left\{\sum_{l=1}^{I_{z}} p_{l}+2 \sum_{l=I_{z}+1}^{T_{z}} p_{l}\right\}+1=\sum_{l=1}^{I} m_{l}+2 \sum_{l=I+1}^{T} m_{l}
$$

so that there are $I_{z}$ zeros at $\xi_{l}, l=1,2, \ldots, I_{z}$ on the imaginary axis and $M_{z}=T_{z}-I_{z}$ pairs of zeros at $\left(\xi_{l},-\bar{\xi}_{l}\right), l=I_{z}+1, \ldots, T_{z}$ off the imaginary axis.

Note that as $\operatorname{Re} \zeta_{l}$, for a particular $l$ in the range $l=I+1, \ldots, T$, tends to zero, the pair $\left(\zeta_{l},-\bar{\zeta}_{l}\right)$ produce factors $\left(\omega-\zeta_{l}\right)^{2 m_{l}}$ while the two terms in (3.1) merge to produce terms in which the highest power in the denominator is $m_{l}$. Thus, $P$ must contain either a factor $\left(\omega-\xi_{k}\right)^{p_{k}}$ where $\xi_{k}$ is on the imaginary axis, such that $\xi_{k} \rightarrow \zeta_{l}$ as $\operatorname{Re} \zeta_{l}$ tends to zero, or if not on the imaginary axis, then a factor $\left(\omega-\xi_{k}\right)^{p_{k}}\left(\omega+\bar{\xi}_{k}\right)^{p_{k}}$ with this property. In the first case, we must have $p_{k}=m_{l}$ and in the second, $2 p_{k}=m_{l}$.

Branch cut singularities yield integrals over exponentials which, for cuts along the imaginary axis in $\Omega^{(+)}$, gives the standard continuous spectrum form 21. Again, the branch cuts may now be anywhere on $\Omega^{(+)}$, subject to the constraint that $G^{\prime}$ is real. It should be pointed out again that branch cut singularities off the imaginary axis may yield oscillatory behaviour in $G^{\prime}$, combined with relaxation behaviour, due to trigonometric functions mutiplying the exponentials.

When only branch cut singularities exist, we obtain

$$
G^{\prime}(s)=\frac{1}{2 \pi} \int_{C} d(\omega) e^{i \omega s} d \omega, \quad t \in \mathbb{R}^{++} .
$$

The integral over $C$ is defined as starting from the end closest to the origin for each cut; if both are equidistant, then a further criterion must be used. We have

$$
d(\omega)=G_{F}^{+}(\omega)-G_{F}^{\prime-}(\omega)
$$

where $G_{F}^{+}(\omega)$ is the limiting value of $G_{F}^{\prime}(\omega)$ approaching the cut segment with increasing $\arg (\omega)$ from the positive real axis, while $G_{F}^{\prime-}(\omega)$ is the limit from the other side. The condition (2.13) imposes constraints on $d(\omega)$.

We exclude these from consideration because, as shown in Section 5 , they lead to a situation where minimal states are singletons, the maximum free energy is the work function and the lower bound on the dissipation is the weakest possible estimate, namely zero.

Essential singularities at infinity of a certain kind are associated with finite memory, i.e. where $G^{\prime}(s)$, or a term in this function, vanishes for $s>s_{d}>0$, the quantity $s_{d}$ being the duration of the memory [19]. For simplicity, we exclude such singularities in this work. Finite memory behaviour can generally be approximated by suitable exponential decay terms.

Essential singularities at finite points on $\Omega^{(+)}$are the remaining possibility. It is difficult to imagine a choice of relaxation behaviour that would generate such behaviour in $G_{F}^{\prime}$. Such singularities are excluded from consideration in this work.

4. Materials with only isolated singularities. We seek the general form of $H(\omega)$ given by (2.14), when $G_{F}^{\prime}$ is a rational function of $\omega$ obeying (2.13). Clearly, $H$ will be 
a real, rational function of $\omega^{2}$ obeying (2.16), so we write

$$
H(\omega)=\frac{N(\omega)}{D(\omega)}
$$

where

$$
\begin{aligned}
D(\omega) & =|Q(\omega)|^{2} \\
& =\prod_{l=1}^{I}\left(\omega^{2}+\left|\zeta_{l}\right|^{2}\right)^{m_{l}} \prod_{l=I+1}^{T}\left[\left(\omega^{2}-\zeta_{l}^{2}\right)\left(\omega^{2}-\bar{\zeta}_{l}^{2}\right)\right]^{m_{l}} \\
& =\prod_{l=1}^{I}\left(\omega^{2}+\left|\zeta_{l}\right|^{2}\right)^{m_{l}} \prod_{l=I+1}^{T}\left[\left(\omega^{2}+\left|\zeta_{l}\right|^{2}\right)^{2}-4 \omega^{2}\left(\operatorname{Re} \zeta_{l}\right)^{2}\right]^{m_{l}} \\
\zeta_{l}=i \alpha_{l}, & \alpha_{l} \in \mathbb{R}^{++}, \quad l=1,2, \ldots, I, \quad \zeta_{l} \in \Omega^{(+)},\left|\operatorname{Re} \zeta_{l}\right|>0, l=I+1, \ldots, T
\end{aligned}
$$

and

$$
\begin{aligned}
N(\omega) & =\frac{\omega}{2 i}\{P(\omega) \bar{Q}(\omega)-\bar{P}(\omega) Q(\omega)\} \\
& =H_{\infty} \prod_{l=1}^{I_{h}}\left(\omega^{2}+\left|\eta_{l}\right|^{2}\right)^{n_{l}} \prod_{l=I_{h}+1}^{T_{h}}\left[\left(\omega^{2}-\eta_{l}^{2}\right)\left(\omega^{2}-\bar{\eta}_{l}^{2}\right)\right]^{n_{l}} \\
& =H_{\infty} \prod_{l=1}^{I_{h}}\left(\omega^{2}+\left|\eta_{l}\right|^{2}\right)^{n_{l}} \prod_{l=I_{h}+1}^{T_{h}}\left[\left(\omega^{2}+\left|\eta_{l}\right|^{2}\right)^{2}-4 \omega^{2}\left(R e \eta_{l}\right)^{2}\right]^{n_{l}} \\
\eta_{1} & =0, n_{1}=1, \eta_{l}=i \gamma_{l}, \gamma_{l} \in \mathbb{R}^{++}, l=2,3, \ldots, I_{h}, \\
& \eta_{l} \in \Omega^{(+)},\left|\operatorname{Re} \eta_{l}\right|>0, l=I_{h}+1, \ldots, T_{h},
\end{aligned}
$$

where

$$
\sum_{l=1}^{I} m_{l}+2 \sum_{l=I+1}^{T} m_{l}=\sum_{l=1}^{I_{h}} n_{l}+2 \sum_{l=I_{h}+1}^{T_{h}} n_{l} .
$$

We observe, as argued earlier, that, for each $l>I+1$, if $\operatorname{Re} \zeta_{l} \rightarrow 0$, then there must be a zero, or a pair of zeros, which cancels one of these singularities on the imaginary axis.

The factors of $H$ given by (2.15) can be deduced by inspection from (4.1) and (4.2). We have for $\omega \in \mathbb{R}$

$$
\begin{aligned}
H_{+}(\omega) & =\frac{N_{+}(\omega)}{D_{+}(\omega)}, \\
N_{+}(\omega) & =h_{\infty} \prod_{l=1}^{I_{h}}\left(\omega-\eta_{l}\right)^{n_{l}} \prod_{l=I_{h}+1}^{T_{h}}\left[\left(\omega-\eta_{l}\right)\left(\omega+\bar{\eta}_{l}\right)\right]^{n_{l}}, \\
D_{+}(\omega) & =\prod_{l=1}^{I}\left(\omega-\zeta_{l}\right)^{m_{l}} \prod_{l=I+1}^{T}\left[\left(\omega-\zeta_{l}\right)\left(\omega+\bar{\zeta}_{l}\right)\right]^{m_{l}}, \\
H_{-}(\omega) & =\bar{H}_{+}(\omega)=(-1)^{c} H_{+}(-\omega), \quad h_{\infty}=H_{\infty}^{1 / 2}, \\
c & =\sum_{l=1}^{I_{h}} n_{l}-\sum_{l=1}^{I} m_{l} .
\end{aligned}
$$


It is clear from (4.3) that $c$ is even so that this facter can be ignored. For our purposes, we can write $H, H_{ \pm}$in simpler and more general forms, since the detailed special structure outlined above is not essential for the developments of the later sections. We put, for $\omega \in \mathbb{R}$, recalling (2.17),

$$
\begin{aligned}
H_{+}(\omega) & =h_{\infty} \frac{\prod_{l=1}^{Z}\left(\omega-\eta_{l}\right)^{n_{l}}}{\prod_{l=1}^{S}\left(\omega-\zeta_{l}\right)^{m_{l}}}, \\
\zeta_{l} & \in \Omega^{(+)}, n_{1}=1, \eta_{1}=0, \quad \eta_{l} \in \Omega^{(+)}, l=2,3, \ldots, Z, \\
H_{-}(\omega) & =\bar{H}_{+}(\omega)=H_{+}(-\omega), \\
H(\omega) & =H_{\infty} \frac{\prod_{l=1}^{Z}\left[\left(\omega-\eta_{l}\right)\left(\omega-\bar{\eta}_{l}\right)\right]^{n_{l}}}{\prod_{l=1}^{S}\left[\left(\omega-\zeta_{l}\right)\left(\omega-\bar{\zeta}_{l}\right)\right]^{m_{l}}}
\end{aligned}
$$

where

$$
\sum_{l=1}^{Z} n_{l}=\sum_{l=1}^{S} m_{l}=q .
$$

We must have for each $\eta_{i}$ an $\eta_{j}$ such that

$$
\bar{\eta}_{i}=-\eta_{j}, \quad n_{i}=n_{j}
$$

If the zero is on the imaginary axis, then $i=j$. A similar statement applies to all $\zeta_{l}$ and $m_{l}$.

We consider a much larger class of factorizations of $H$ obtained by interchanging the zeros of $H_{+}$and $H_{-}$, excluding of course the simple zero at the origin. Such interchanges leave the singularity structure unchanged. Noting (4.5), we see that there are

$$
N=2^{p}, \quad p=\sum_{l=2}^{Z} n_{l}=q-1,
$$

distinct factorizations of this kind which we distinguish by the label $f=1,2, \ldots, N$. The case $f=1$ is where no zeros are interchanged and the case $f=N$ is where all zeros are interchanged. For real $\omega$, these can be written as

$$
\begin{aligned}
H(\omega) & =H_{+}^{f}(\omega) H_{-}^{f}(\omega), \\
H_{+}^{f}(\omega) & =h_{\infty} \frac{\prod_{l=1}^{Z}\left(\omega-\eta_{l}\right)^{a_{l}^{f}}\left(\omega-\bar{\eta}_{l}\right)^{b_{l}^{f}}}{\prod_{l=1}^{S}\left(\omega-\zeta_{l}\right)^{m_{l}}}, \\
H_{-}^{f}(\omega) & =\bar{H}_{+}^{f}(\omega), \\
a_{l}^{f}+b_{l}^{f} & =n_{l}, l=2,3, \ldots, Z, \quad a_{l}^{f}, b_{l}^{f} \geq 0 .
\end{aligned}
$$

Note that the permutation $f$ is completely specified by the $Z$-1-dimensional vector $a_{l}^{f}, l=2,3, \ldots, Z$.

Most of the relations required later do not depend in a vital way on the location (i.e., whether in the upper or the lower complex plane) of the zeros of $H_{+}$and $H_{-}$, but they do depend crucially on the location of the singularities.

We can consider the different factorizations in the following way. The quantity

$$
X_{l}(\omega)=\frac{\omega-\bar{\eta}_{l}}{\omega-\eta_{l}}
$$


for real $\omega$ is a phase transformation. Then

$$
H_{+}^{f}(\omega)=\left\{\prod_{l=2}^{Z} X_{l}^{b_{l}^{f}}(\omega)\right\} H_{+}(\omega) .
$$

The corresponding $H_{-}^{f}$ is obtained by taking the complex conjugate.

Note that if one of a pair of zeros related by (4.6) is interchanged but not the other, then we no longer have the property

$$
\bar{H}_{ \pm}^{f}(\omega)=H_{ \pm}^{f}(-\omega) .
$$

The developments from here to the end of the section are closely related to corresponding material in [17. We first present certain results to be used later which can be proved in a manner similar to that given in [14], but with the relative strain $E_{r}^{t}$ replacing $E^{t}$.

Relations (2.32) and (2.33) can be generalized to different factorizations. We have

$$
\begin{aligned}
P^{(f t)}(\omega) & =H_{-}^{f}(\omega) E_{r+}^{t}(\omega) \\
& =p_{-}^{(f t)}(\omega)-p_{+}^{(f t)}(\omega)
\end{aligned}
$$

where $p_{-}^{(f t)}(\omega)$ is analytic in $\Omega^{+}$, going to zero at large $\omega$ as strongly as $\omega^{-1}$, while $p_{+}^{(f t)}(\omega)$ is analytic in $\Omega^{-}$with similar large $\omega$ behaviour. They are given by the limit $z \rightarrow \omega \in \mathbb{R}$ of

$$
p^{(f t)}(z)=\frac{1}{2 \pi i} \int_{-\infty}^{\infty} \frac{P^{(f t)}(\omega)}{\omega-z} d \omega
$$

from above and below the real axis, respectively. The analyticity of these quantities on $\mathbb{R}$ follows from the assumed analyticity of $H_{-}^{f}$ and $E_{+}^{t}$ on $\mathbb{R}[14]$. They are defined over the entire complex plane by analytic continuation. If (4.10) holds, we have

$$
\bar{p}_{ \pm}^{(f t)}(\omega)=p_{ \pm}^{(f t)}(-\omega), \quad \omega \in \mathbb{R}
$$

but otherwise this need not be true.

Using (2.22), it can be shown that [19] 30]

$$
\begin{aligned}
\frac{d}{d t} p_{+}^{(f t)}(\omega) & =-i \omega p_{+}^{(f t)}(\omega)-K_{f}(t), \\
\frac{d}{d t} p_{-}^{(f t)}(\omega) & =-i \omega p_{-}^{(f t)}(\omega)-K_{f}(t)-\frac{H_{-}^{f}(\omega) \dot{E}(t)}{i \omega}, \\
K_{f}(t) & =\frac{1}{2 \pi} \int_{-\infty}^{\infty} H_{-}^{f}(\omega) E_{r+}^{t}(\omega) d \omega .
\end{aligned}
$$

The quantity $K_{f}(t)$ is not always a real quantity because of the failure of property (4.10). Also

$$
\lim _{|\omega| \rightarrow \infty} \omega p_{ \pm}^{(f t)}(\omega)=i K_{f}(t)
$$

and

$$
\frac{1}{2 \pi} \int_{-\infty}^{\infty} p_{ \pm}^{(f t)}(\omega) d \omega=\mp \frac{1}{2} K_{f}(t) .
$$

Let $E^{t}$ be a given history with constant continuation, i.e. $E^{t}(s)=E(t), s \in \mathbb{R}^{--}$. Let

$$
E_{d}^{t}=E_{1}^{t}-E^{t}=E_{r 1}^{t}-E_{r}^{t}
$$


where in general $E_{r 1}^{t}, E_{d}^{t}$ are not assumed to vanish on $\mathbb{R}^{--}$. The second relation is in terms of relative histories. The constraint

$$
\int_{-\infty}^{\infty} G_{2}(|s-u|) E_{d}^{t}(u) d u=0, \quad s \in \mathbb{R}^{-},
$$

is imposed, where the subscript ' 2 ' indicates differentiation with respect to $u$, giving a discontinuous function. If $E_{r 1}^{t}$ vanishes on $\mathbb{R}^{++}$, then (4.16) becomes

$$
\begin{gathered}
\int_{-\infty}^{0} G_{2}(|s-u|) E_{m}^{t}(u) d u=-\int_{0}^{\infty} G_{2}(u-s) E_{r}^{t}(u) d u, \\
E_{m}^{t}(u)=-E_{1}^{t}(u), \quad s \in \mathbb{R}^{-},
\end{gathered}
$$

which is the Wiener-Hopf equation for $E_{m}^{t}$, the optimal continuation which yields the maximum recoverable work (17], for example). In fact, the solution of this equation is the quantity given by (2.35).

If $E_{r 1}^{t}$ vanishes on $\mathbb{R}^{--}$, then (4.16) becomes

$$
\int_{0}^{\infty} G^{\prime}(u+|s|) E_{d}^{t}(u) d u=0, \quad s \in \mathbb{R}^{-}
$$

which, with the condition $E(t)=E_{1}(t)$, implies that $E_{r}^{t}$ and $E_{r 1}^{t}$ are in the same minimal state, as defined by (2.4).

We consider a generalization of (4.11) where the strain is defined over $\mathbb{R}$, with Fourier transforms given by $(2.20) 1$. With a view to future applications, the relevant relations will be written for $E_{d}^{t}$,

$$
\begin{aligned}
U^{(f t)}(\omega) & =H_{-}^{f}(\omega) E_{d F}^{t}(\omega)=H_{-}^{f}(\omega)\left(E_{d+}^{t}(\omega)+E_{d-}^{t}(\omega)\right) \\
& =u_{-}^{(f t)}(\omega)-u_{+}^{(f t)}(\omega)
\end{aligned}
$$

where

$$
\begin{aligned}
u_{ \pm}^{(f t)}(\omega) & =\lim _{z \rightarrow \omega \mp} u^{(f t)}(z), \\
u^{(f t)}(z) & =\frac{1}{2 \pi i} \int_{-\infty}^{\infty} \frac{U^{(f t)}(\omega)}{\omega-z} d \omega .
\end{aligned}
$$

The quantities $u_{ \pm}^{(f t)}$ have the same analyticity properties as $p_{ \pm}^{(f t)}$. It is clear that

$$
\begin{aligned}
& u_{-}^{(f t)}(\omega)=p_{d-}^{(f t)}+H_{-}^{f}(\omega) E_{d-}^{t}(\omega)=p_{d+}^{(f t)}+H_{-}^{f}(\omega) E_{d F}^{t}(\omega), \\
& u_{+}^{(f t)}(\omega)=p_{d+}^{(f t)}
\end{aligned}
$$

where $p_{d \pm}^{(f t)}$ are defined by (4.11) and (4.12) with $E_{d+}^{t}$ replacing $E_{r+}^{t}$.

As in (4.21), we will use the symbol $u$ when the relevant strain is defined over $\mathbb{R}$ and $p$ when it is defined over $\mathbb{R}^{+}$.

We will now prove results which are not confined to a material with only isolated singularities. They rely upon the fact that $H(\omega)$ is factorizable into quantities analytic on $\Omega^{+}$and $\Omega^{-}$, with zeros that may be interchanged. Analyticity excludes non-isolated (i.e. branch cut) as well as isolated singularities, of course. The results rely also on the analyticity of the various quantities on the real axis. 
Proposition 4.1. The strain history and continuation $E_{d}^{t}$ obey 4.16$)$ if $u_{-}^{(f t)}$ vanishes. Relation (4.16) implies that $u_{-}^{(f t)}$ vanishes in two cases: (i) where no exchange of zeros in $H_{ \pm}$takes place $(f=1)$ and (ii) where $E_{d-}^{t}$ vanishes.

Proof. Applying Plancherel's theorem to (4.16) and using (2.24) gives

$$
\int_{-\infty}^{\infty} \frac{H(\omega)}{\omega}\left(E_{d+}^{t}(\omega)+E_{d-}^{t}(\omega)\right) e^{i \omega s} d \omega=0, \quad s \in \mathbb{R}^{-} .
$$

With the aid of (4.19), we can write (4.22) in the form

$$
\int_{-\infty}^{\infty} \frac{H_{+}^{f}(\omega)}{\omega}\left(u_{-}^{(f t)}(\omega)-u_{+}^{(f t)}(\omega)\right) e^{i \omega s} d \omega=0, \quad s \in \mathbb{R}^{-},
$$

where $H_{+}^{f}(\omega)$ vanishes linearly at $\omega=0$. Closing the integral involving $u_{+}^{(f t)}$ on $\Omega^{-}$, we see that it vanishes by Cauchy's theorem, so that the condition becomes (cf. (2.39))

$$
F(s)=\int_{-\infty}^{\infty} \frac{H_{+}^{f}(\omega)}{\omega} u_{-}^{(f t)}(\omega) e^{i \omega s} d \omega=0, \quad s \in \mathbb{R}^{-},
$$

clearly satisfied if $u_{-}^{(f t)}$ vanishes, which is the first assertion. We now prove that, in the two specified cases, if (4.23) is satisfied, then $u_{-}^{(f t)}$ is zero. The function $F(s)$ vanishes on $\mathbb{R}^{-}$, so that its Fourier transform $2 \pi\left(H_{+}^{f}(\omega) / \omega\right) u_{-}^{(f t)}(\omega)$ must be analytic in $\Omega^{(-)}$. This can only be true if $H_{+}^{f}(\omega) / \omega$ vanishes at each of the isolated singularities of $u_{-}^{(f t)}$ in $\Omega^{(-)}$; and for each cut type singularity in $u_{-}^{(f t)}$, we must have a compensating singularity of the same kind in $H_{+}^{f}(\omega) / \omega$. Since $H_{+}^{f}(\omega) / \omega$ is analytic in $\Omega^{(-)}, u_{-}^{(f t)}$ cannot have cuts in this half-plane. As for isolated singularities, we distinguish the case where no permutation of zeros has taken place $(f=1)$, or case(i), from all others.

For case (i) or $f=1, H_{+}$has no zeros in $\Omega^{(-)}$. The only remaining option is that $u_{-}^{(f t)}$ has no singularities in $\Omega^{(-)}$and therefore in $\Omega$. It vanishes at infinity as $\omega^{-1}$, and therefore, by Liouville's theorem, vanishes everywhere.

If permutations of zeros are allowed, then $H_{+}^{f}$ has zeros in $\Omega^{(-)}$. Consider the definition of $u_{-}^{(f t)}$, given by (4.19) and 4.20). Evaluating the integral by closing the contour on $\Omega^{(-)}$shows that $u_{-}^{(f t)}$ has singularities in $\Omega^{(-)}$where $H_{-}^{f}$ and $E_{d-}^{t}$ have singularities, and these must be isolated, as concluded above. If a zero in $H_{+}^{f}$ cancels a singularity in $H_{-}^{f}$, then, without permutation, the zero and the singularity would cancel in $H$. In other words, there would be no singularity or zero to begin with. Therefore, the only option is that $u_{-}^{(f t)}$ is free of singularities due to $H_{-}^{f}$. It could still have singularities due to $E_{d-}^{t}(\omega)$ cancelled by zeros in $H_{+}^{f}$. However, if $E_{d-}^{t}$ is zero, which is case(ii), this cannot be so. Thus, by Liouville's theorem, $u_{-}^{(f t)}$ must be zero.

It will become clear in Section 7 that the first case $(f=1)$ corresponds to the solution of (4.17), yielding the minimum free energy, given by (2.36), while, from an argument given in Section 5 case (ii) corresponds to the maximum free energy $(f=N)$. In fact we see from (4.21) that $E_{d-}^{t}$ vanishing yields $p_{d-}^{(N t)}=0$ so that Proposition 4.1 states that condition (4.16) or (4.18) in this case implies that $p_{d-}^{(N t)}$ vanishes. Recalling that (4.18) 
is the condition for equivalence of states $E_{1}^{t}$ and $E^{t}$, we conclude that $p_{1-}^{(N t)}$ defined by (4.11), for a given history $E_{1}^{t}$, is a minimal state variable.

In Section[7, we shall adopt the condition $u_{-}^{(f t)}=0$ as the constraint on our variational scheme in all cases, not just in the two described in Proposition 4.1 This condition implies (4.16) but is a stronger assumption in general - except in the two cases - which is in effect the content of Proposition 4.1

Observe that by applying the argument which yields (4.23) to (2.23), using (4.11), the stress function at time $t$ can be written as (see (2.39) $)$

$$
T(t)=G_{\infty} E(t)+\frac{1}{\pi i} \int_{-\infty}^{\infty} \frac{H_{+}^{f}(\omega)}{\omega} p_{-}^{(f t)}(\omega) d \omega
$$

for each permutation $f$. All of these are of course equivalent. It is proved in [13, for general materials and in the full tensorial case, that (stated for scalars) $p_{-}^{(f t)}$ is a minimal state variable for $f=1$. The argument used in that reference can easily be extended to all $f$. Briefly, it amounts to showing that if $F(s)$, given by (4.23), but with $u_{-}^{(f t)}$ replaced by $p_{d-}^{(f t)}\left(E_{d-}^{t}\right.$ zero or $E_{r 1}^{t}$ vanishing on $\mathbb{R}^{-}$, yielding (4.18) $)$vanishes on $\mathbb{R}^{-}$, then $p_{d-}^{(f t)}$ is zero. This follows by the argument used in case (ii) of Proposition 4.1. Thus, we state

Corollary 4.2. The quantity $p_{-}^{(f t)}$ is a function of the minimal state for all permutations $f$.

If $p_{-}^{(f t)}$ is a function of state, then in general $p_{+}^{(f t)}=p_{-}^{(f t)}-H_{-}^{f} E_{r+}^{t}$ will not be, because of the occurrence of the transformed history.

It is convenient to introduce a conventional scalar product

$$
\langle f, g\rangle=\frac{1}{2 \pi} \int_{-\infty}^{\infty} \bar{f}(\omega) g(\omega) d \omega .
$$

Observe that $f$ and $g$ are orthogonal in this scalar product if $f$ is analytic in $\Omega^{+}$and $g$ in $\Omega^{-}$or vica versa and both vanish at infinity at least as strongly as $\omega^{-1}$; see for example (2.34).

We write

$$
\langle f, f\rangle=\|f\|_{L}^{2}
$$

indicating the Lesbeque $L^{2}$ norm. Also, we will use the norm

$$
\left\|E_{r}^{t}\right\|^{2}=\frac{1}{2 \pi} \int_{-\infty}^{\infty} H(\omega)\left|E_{r+}^{t}(\omega)\right|^{2} d \omega .
$$

5. The maximum free energy. We now consider the maximum free energy of a given state, defined as the minimum energy required to achieve this state (for example [4] 5, 10, 17, 20]) from the zero state. There are two distinct cases here: (1) where the maximum free energy is equal to the work function; this occurs when the set of minimal states, defined by (2.4), is a singleton, with certain exceptions - see the remark after Proposition 5.1 below; and (2) when it is less than the work function, which is true in the case of materials for which the space of minimal states contains more than one member. 
It was shown in [17, 20, that the latter situation prevails for discrete spectrum materials (the relaxation function given by a sum of strictly decaying exponentials), by deriving and solving a Weiner-Hopf equation, subject to a constraint enforced by a Lagrange multiplier function. A simpler and more general argument is developed here.

For continuous spectrum materials (the relaxation function given by an integral of a density function, with some continuity characteristics, multiplying a strictly decaying exponential), it is shown in 21] that the first situation applies.

A difference between isolated and branch cut singularities is that the former always have infinite behaviour associated with them, while the latter are characterized by generally finite discontinuities, though in fact, infinities may occur at branch points and indeed on the cut. However, there is the following clear-cut distinction which is important in the present context.

REMARK 5.1. If a function $F$ defined on $\Omega$ has isolated singularities at a set of points, then $1 / F$ will have zeros at these points, while if $F$ has a branch cut between two branch points, then $1 / F$ will also have a branch cut between these two branch points. The converse of these two statements also holds.

We consider a material characterized by isolated singularities or branch cuts or both and a factorization where zeros, if present, may be interchanged. We will use the notation of (4.8) 1 but will not initially assume the form $(4.8)_{2}$.

Let us write (2.4) in the following way: let $\left(E(t), E_{r}^{t}\right)$ be a given current strain, strain history couple. Then $\left(E_{1}(t), E_{1}^{t}\right)$ is in the same minimal state as $\left(E(t), E_{r}^{t}\right)$ if

$$
E_{d}(t)=0, \quad \int_{0}^{\infty} \frac{\partial}{\partial s} G(|s-u|) E_{d}^{t}(u) d u=0, \quad s \in \mathbb{R}^{-},
$$

where $E_{d}(t)=E_{1}(t)-E(t), E_{d}^{t}(u)=E_{r 1}^{t}(u)-E_{r}^{t}(u)$. We write this in the form $(c f$. (4.18) )

$$
\begin{aligned}
\int_{0}^{\infty} \frac{\partial}{\partial s} G(|s-u|) E_{d}^{t}(u) d u & =R(s), \quad s \in \mathbb{R}^{++}, \\
& =0, \quad s \in \mathbb{R}^{-}
\end{aligned}
$$

and we take Fourier transforms to obtain

$$
\frac{2 i}{\omega} H(\omega) E_{d+}^{t}(\omega)=R_{+}(\omega),
$$

using (2.24). Equations (4.8) 1 and (4.11) can now be utilized to write this in the form

$$
\frac{2 i}{\omega}\left[H_{+}^{f}(\omega)\left(p_{d-}^{(f t)}(\omega)-p_{d+}^{(f t)}(\omega)\right)\right]=R_{+}(\omega) .
$$

It follows from Corollary 4.2 that

$$
p_{d-}^{(f t)}(\omega)=0
$$

and we have

$$
R_{+}(\omega)=-\frac{2 i}{\omega} H_{+}^{f}(\omega) p_{d+}^{(f t)}(\omega) .
$$


Then, by virtue of (5.1),

$$
E_{d+}^{t}(\omega)=-\frac{1}{H_{-}^{f}(\omega)} p_{d+}^{(f t)}(\omega)
$$

Now $E_{d+}^{t}$, if it is non-zero, must be analytic in $\Omega^{-}$. Therefore, $H_{-}^{f}$ can have no branch cut singularities and we adopt the form $(4.8)_{2}$. It follows from $(4.8)_{1}$ and $(4.8)_{3}$ that $H_{+}^{f}$ and $H$ have the same property. Also, the zeros of $H_{-}^{f}(\omega)$ must be in $\Omega^{(+)}$which means that the factorization must be associated with $f=N$, namely where all the zeros are interchanged. Thus we have

Proposition 5.1. For a material where $G_{F}^{\prime}$ has no essential singularities, the set of minimal states has more than one member only if $G_{F}^{\prime}$ possesses no branch cut singularities.

In other words, $G_{F}^{\prime}$ can have only isolated singularities. Even if this is true, there are special cases - notably one simple pole, corresponding to a single exponential in $G^{\prime}$ where the set of minimal states may still be a singleton [12]. In this case, the minimum and maximum free energies are equal and both are less than the work function; see (9.15) below.

If $G_{F}^{\prime}$ has branch cut singularities, then the set of minimum states is a singleton, $E_{d}^{t}$ is zero and the work function is the maximum free energy. In this case, the state is defined by $\left(E(t), E_{r}^{t}\right)$ and the work function is a function of state.

We now seek the choice of state $\left(E_{1}(t), E_{r 1}^{t}\right)$ such that the work done to achieve this state is least among members of the minimal state which has $\left(E(t), E_{r}^{t}\right)$ as a member. We have from (2.27) and (2.34)

$$
\begin{aligned}
\widetilde{W}\left(E_{1}(t), E_{1}^{t}\right) & =W_{1}(t)=\phi(t)+\frac{1}{2 \pi} \int_{-\infty}^{\infty} H(\omega)\left|E_{r 1+}^{t}(\omega)\right|^{2} d \omega \\
& =\phi(t)+\frac{1}{2 \pi} \int_{-\infty}^{\infty}\left[\left|p_{1-}^{t}(\omega)\right|^{2}+\left|p_{1+}^{t}(\omega)\right|^{2}\right] d \omega
\end{aligned}
$$

where

$$
p_{1 \pm}^{t}(\omega)=\frac{1}{2 \pi i} \int_{-\infty}^{\infty} \frac{H_{-}^{N}\left(\omega^{\prime}\right) E_{r 1+}^{t}\left(\omega^{\prime}\right) d \omega^{\prime}}{\omega^{\prime}-\omega^{\mp}} .
$$

Now, recalling (5.2), we see $p_{1-}^{t}$ is fixed by virtue of the fact that it is equal to $p_{-}^{(N t)}$, defined by (5.4) but with $E_{r 1+}^{t}$ replaced by $E_{r+}^{t}$. However, $p_{1+}^{t}$ can be varied and the choice which minimizes $W_{1}$ is clearly

$$
p_{m+}^{t}(\omega)=0, \omega \in \mathbb{R}
$$

where $p_{m+}^{t}$ is the optimal choice, corresponding to an optimal history $E_{m}^{t}$. Noting that

$$
p_{d+}^{t}(\omega)=p_{m+}^{t}(\omega)-p_{+}^{(N t)}(\omega)=-p_{+}^{(N t)}(\omega)
$$

where $p_{+}^{(N t)}$ is given by (5.4) with $E_{r 1+}^{t}$ replaced by $E_{r+}^{t}$, we see that (5.3) gives 


$$
\begin{aligned}
E_{m+}^{t}(\omega) & =E_{r+}^{t}(\omega)+\frac{1}{H_{-}^{N}(\omega)} p_{+}^{(N t)}(\omega) \\
& \left.=\frac{1}{H_{-}^{N}(\omega)}\left\{p_{-}^{(N t)}(\omega)-p_{+}^{(N t)}(\omega)+p_{+}^{(N t)}(\omega)\right)\right\} \\
& =\frac{p_{-}^{(N t)}(\omega)}{H_{-}^{N}(\omega)} .
\end{aligned}
$$

Proposition 5.2. For materials where $G_{F}^{\prime}$ has only isolated singularities, the maximum free energy is given by

$$
\psi_{M}(t)=\phi(t)+\frac{1}{2 \pi} \int_{-\infty}^{\infty}\left|p_{-}^{(N t)}(\omega)\right|^{2} d \omega
$$

and the Fourier transformed optimal history associated with this quantity has the form

$$
E_{m+}^{t}(\omega)=\frac{p_{-}^{(N t)}(\omega)}{H_{-}^{N}(\omega)}
$$

where

$$
p_{-}^{(N t)}(\omega)=\frac{1}{2 \pi i} \int_{-\infty}^{\infty} \frac{H_{-}^{N}\left(\omega^{\prime}\right) E_{+}^{t}\left(\omega^{\prime}\right) d \omega^{\prime}}{\omega^{\prime}-\omega^{+}} .
$$

The associated rate of dissipation is given by

$$
D_{M}(t)=\left|K_{M}(t)\right|^{2}, \quad K_{M}(t)=\frac{1}{2 \pi} \int_{-\infty}^{\infty} H_{-}^{N}(\omega) E_{r+}^{t}(\omega) d \omega .
$$

If $G_{F}^{\prime}$ has branch cut singularities, then the maximum free energy is equal to the work function.

Relations (5.8) may be shown using (2.31), (4.13) and (4.15). This, in effect, expresses property P4 or 2.31) of the Graffi conditions. Properties P2 and P3 follow immediately from (5.6) while P1 may be demonstrated in a manner similar to that applied to (2.36), noting (2.38), (2.39) and (4.24).

6. Free energies as discrete quadratic forms. We write the constitutive equation (2.1) 2 for $G^{\prime}$ given by (3.2) in the form

$$
\begin{aligned}
T(t) & =G_{\infty} E(t)+\sum_{l=0}^{S} \sum_{j=0}^{m_{l}-1}(i)^{j} c_{j}^{l} E_{r+}^{t}\left(-\zeta_{l}, j\right) \\
& =G_{\infty} E(t)+\sum_{l=0}^{S} \sum_{j=0}^{m_{l}-1}(i)^{j} c_{j}^{l} E_{r+}^{t}\left(\bar{\zeta}_{l}, j\right), \\
E_{r+}^{t}(\omega, j) & =\frac{d^{j}}{d \omega^{j}} E_{r+}^{t}(\omega), \quad \overline{E_{r+}^{t}\left(-\zeta_{l}, j\right)}=(-1)^{j} E_{r+}^{t}\left(\bar{\zeta}_{l}, j\right) .
\end{aligned}
$$

The coefficients $c_{j}^{l}$ are real. Substituting $G^{\prime}$ given by (3.2) into (2.4), we see that two states are equivalent if and only if the difference between the current strains and histories, 
denoted by $\left(E_{d}(t), E_{d}^{t}\right)$, has the properties outlined in the following. We have

$$
\begin{aligned}
G^{\prime}(s+\tau) & =\sum_{l=1}^{S} g_{l}(s+\tau) e^{i \zeta_{l}(s+\tau)} \\
& =\sum_{l=1}^{S} \sum_{j=0}^{m_{l}-1} \sum_{k=0}^{j} L_{j k}^{l} s^{j-k} \tau^{k} e^{i \zeta_{l}(s+\tau)} \\
& =\sum_{l=1}^{S} \sum_{k=0}^{m_{l}-1} \sum_{j=k}^{m_{l}-1} L_{j k}^{l} s^{j-k} \tau^{k} e^{i \zeta_{l}(s+\tau),} \\
L_{j k}^{l} & ={ }^{j} \mathbf{C}_{k} c_{j}^{l}
\end{aligned}
$$

where ${ }^{j} \mathbf{C}_{k}$ is the standard combinatorial quantity defined by

$$
{ }^{j} \mathbf{C}_{k}=\frac{j !}{(j-k) ! k !} .
$$

The arbitrariness of the factor $e^{i \omega \tau}$ allows us to put each individual term in the summation over singularities to zero. Then, equating the coefficient of each power of $\tau$ to zero, we obtain

$$
\begin{aligned}
E_{d}(t) & =0 \\
\int_{0}^{\infty} \sum_{j=k}^{m_{l}-1} L_{j k}^{l} s^{j-k} e^{i \zeta_{l} s} E_{d}^{t}(s) d s & =\sum_{j=k}^{m_{l}-1} L_{j k}^{l}(i)^{j-k} E_{d+}^{t}\left(-\zeta_{l}, j-k\right)=0, \\
k=0,1, \ldots, m_{l}-1, \quad l & =1,2, \ldots, S, \\
E_{d+}^{t}(\omega, k) & =\frac{d^{k}}{d \omega^{k}} E_{d+}^{t}(\omega) .
\end{aligned}
$$

Taking $k=m_{l}-1, m_{l}-2, \ldots$, we deduce that $E_{d+}\left(-\zeta_{l}, 0\right)$ vanishes if $L_{\left(m_{l}-1\right)\left(m_{l}-1\right)}^{l} \neq 0$ while $E_{d+}\left(-\zeta_{l}, 1\right)$ vanishes if $L_{\left(m_{l}-1\right)\left(m_{l}-2\right)}^{l} \neq 0$ and so on. Now $L_{\left(m_{l}-1\right) k}^{l}$ will vanish only if $c_{m_{l}-1}^{l}$ is zero. However, as noted after (3.2), $r_{l m_{l}}$ will always be non-zero so that $c_{m_{l}-1}^{l}$ will never vanish, by virtue of $(3.2)_{3}$. Thus

$$
\begin{aligned}
& E_{d}(t)=0, \quad E_{d+}^{t}\left(-\zeta_{l}, j\right)=0, \\
& j=0,1, \ldots, m_{l}-1, \quad l=1,2, \ldots, S .
\end{aligned}
$$

Define a vector e in $\mathbb{R}^{S} \times \mathbb{R}^{m}, m=\max \left\{m_{l}, l=1,2, \ldots, S\right\}$ with components

$$
\begin{aligned}
& e_{l j}(t)=\frac{1}{Z_{l j}} E_{r+}^{t}\left(-\zeta_{l}, j\right), \quad Z_{l j}=-\frac{i j !}{\zeta_{l}^{j+1}}, \\
& \bar{e}_{l j}(t)=\frac{(-1)^{j}}{\bar{Z}_{l j}} E_{r+}^{t}\left(\bar{\zeta}_{l}, j\right), \quad j=0,1, \ldots, m-1, \quad l=1,2, \ldots, S .
\end{aligned}
$$

Consider the quantity

$$
\psi(t)=\phi(t)+\frac{1}{2} \mathbf{e}^{*} \mathbf{C e}
$$

where $\phi(t)$ is the elastic free energy defined by $(2.26)_{3}$ and $\mathbf{C}$ is a hermitean, positive definite matrix with components $C_{n k l j}, n, l=1,2, \ldots, S, j, k=0,1, \ldots, m-1$. It is 
understood that

$$
C_{n k l j}=0, \quad \text { if } k \geq m_{n} \text { or } j \geq m_{l} .
$$

It is clear that $\psi(t)$ has property $\mathrm{P} 3$, given by (2.30), of a free energy. For a stationary history $E^{t}(s)=E(t), s \in \mathbb{R}^{+}$, we have $e_{l j}(t)=0, l=1,2, \ldots, S, j=0,1, \ldots, m_{l}-1$. Therefore, $\psi(t)$ has property P2 or (2.29) of a free energy. Property P1, given by (2.28), requires that

$$
\begin{aligned}
& \sum_{n=1}^{S} \sum_{k=0}^{m_{n}-1} C_{n k l j}=(i)^{j} c_{j}^{l} Z_{l j}, \quad j=0,1, \ldots, m_{l}-1, \quad l=1,2, \ldots, S, \\
& \sum_{l=1}^{S} \sum_{j=0}^{m_{l}-1} C_{n k l j}=(-i)^{k} c_{k}^{n} \bar{Z}_{n k}, \quad j=0,1, \ldots, m_{n}-1, \quad l=1,2, \ldots, S,
\end{aligned}
$$

as one can demonstrate using the relations (6.1) and

$$
\frac{\partial}{\partial E(t)} e_{l j}=\frac{\partial}{\partial E(t)} \bar{e}_{l j}=1, j=0,1, \ldots, m_{l}-1, \quad l=1,2, \ldots, S,
$$

which follow from (6.4) and (2.18). From (2.22), we have

$$
\begin{aligned}
\frac{d}{d t} E_{r+}^{t}(\omega, j) & =-i\left(\frac{\partial}{\partial \omega}\right)^{j}\left[\omega E_{r+}^{t}(\omega)-\frac{\dot{E}(t)}{\omega}\right] \\
& =-i\left[\omega E_{r+}^{t}(\omega, j)+j E_{r+}^{t}(\omega, j-1)-\frac{(-1)^{j} j !}{\omega^{j+1}} \dot{E}(t)\right],
\end{aligned}
$$

where $E_{r+}^{t}(\omega, k)$ vanishes for $k<0$. Using this result, one can show, noting (6.6), that

$$
\begin{aligned}
\dot{\psi}(t)+D(t) & =T(t) \dot{E}(t), \\
D(t) & =\frac{1}{2} \mathbf{e}^{*} \mathbf{\Gamma e}, \\
\Gamma_{n k l j} & =i\left(\bar{\zeta}_{n}-\zeta_{l}\right) C_{n k l j}+i\left[\zeta_{l} C_{n k l(j+1)}-\bar{\zeta}_{n} C_{n(k+1) l j}\right], \\
n, l & =1,2, \ldots, S, \quad j, k=0,1, \ldots, m-1
\end{aligned}
$$

where $\Gamma_{n k l j}$ are the elements of the matrix $\Gamma$. The final requirement $\mathrm{P} 4$, given after (2.31), is that $D(t) \geq 0$ so that $\boldsymbol{\Gamma}$ will be assumed to be positive definite or at worst semi-definite (see Section 8). Thus, under these constraints on C, the quantity $\psi(t)$, given by (6.5), is a free energy.

It follows from (6.3) that free energies of the form (6.5), for a given history, are functions of the minimal state in the sense of $22,12,11$. Such forms of the free energy have been studied by Graffi and Fabrizio [24, 7] and Fabrizio et al. 37, 17, for the case of strictly decaying exponentials.

The forms of $\mathbf{C}$ and $\boldsymbol{\Gamma}$ corresponding to the minimum and maximum free energies can be deduced from (2.36) and (5.6). Other forms are derived in Section 7

Proposition 6.1. The set of free energies expressible in the form (6.5), where $\mathbf{C}$ has the required positivity properties, is identical to the set of free energies associated with the equivalence class containing $\left(E(t), E_{r}^{t}\right)$, which are functions of the minimal state. 
Proof. The set of free energies expressible in the form (6.5), where $\mathbf{C}$ has the required positivity properties, is contained in the set of free energies associated with the equivalence class of $\left(E(t), E_{r}^{t}\right)$, which are functions of the minimal state, since two current value, history pairs $\left(E(t), E_{r}^{t}\right)$ and $\left(E_{1}(t), E_{r 1}^{t}\right)$ in the same minimal state will yield the same value of $\psi(t)$ by virtue of (6.3) . It is also the most general quadratic form that has this property since if we include $E_{r+}^{t}$ and $E_{r 1+}^{t}$ at values of $\omega$ other than $-\zeta_{l}, l=1,2, \ldots, S$, the two quadratic forms will not be equal in general, after imposing (6.3).

This is a convex set, by virtue of a result in 9] and earlier references quoted therein, that any set of free energies, associated with a given history and current value, has this property. The results of the next sections will provide a family of members of this set.

7. A family of free energies that are functions of the minimal state. We will now derive expressions for free energies corresponding to each permutation $f$, introduced after (4.6), and a given state $\left(E(t), E_{r}^{t}\right)$. This family includes the minimum free energy (2.36), the maximum free energy given by (5.6) and intermediate functionals. They are all functionals of the minimal state. The derivation is somewhat simpler than that given in 17 .

The following constrained optimization problem is considered, the aim of which is to yield a free energy. For a given state $\left(E(t), E_{r}^{t}\right)$, we seek $E_{d}^{t}$ which minimizes $\left\|E_{r 1}^{t}\right\|^{2}$, using the notation of (4.27), where $E_{r 1}^{t}=E_{r}^{t}+E_{d}^{t}$, subject to the condition (see (4.20))

$$
u_{-}^{(f t)}(\omega)=\frac{1}{2 \pi i} \int_{-\infty}^{\infty} \frac{H_{-}^{f}\left(\omega^{\prime}\right) E_{d F}^{t}\left(\omega^{\prime}\right)}{\omega^{\prime}-\omega^{+}} d \omega^{\prime}=0 .
$$

Note that in general $E_{r 1}^{t}, E_{d}^{t}$ are non-zero on $\mathbb{R}$ while $E_{r}^{t}$ is taken to be zero on $\mathbb{R}^{-}$. This is stronger than condition (4.16) except in two cases, as indicated by Proposition 4.1.

If the optimal choice of $E_{d}^{t}$ is $E_{d m}^{t}$ so that the corresponding history/continuation $E_{r 1}^{t}$ is $E_{m 1}^{t}=E_{r}^{t}+E_{d m}^{t}$, then the resulting free energy is

$$
\psi_{f}(t)=\phi(t)+\left\|E_{m 1}^{t}\right\|^{2} .
$$

This choice is given some a priori motivation in [17, 20]. However, its justification here will simply be that one can demonstrate that the resulting explicit form has in fact the required properties of a free energy.

It follows from (4.21) that

$$
E_{d F}^{t}(\omega)=-\frac{p_{d+}^{(f t)}(\omega)}{H_{-}^{f}(\omega)}=-\frac{1}{2 \pi i H_{-}^{f}(\omega)} \int_{-\infty}^{\infty} \frac{H_{-}^{f}\left(\omega^{\prime}\right) E_{d+}^{t}\left(\omega^{\prime}\right)}{\omega^{\prime}-\omega^{-}} d \omega^{\prime} .
$$

Using the factorization (4.8), we see that the quantity to be minimized has the form

$$
M(t)=\left\|H_{-}^{f} E_{1}^{t}\right\|_{L}^{2}=\left\|u_{1+}^{(f t)}-u_{1-}^{(f t)}\right\|_{L}^{2}=\left\|u_{1+}^{(f t)}\right\|_{L}^{2}+\left\|u_{1-}^{(f t)}\right\|_{L}^{2}
$$

in the notation of (4.26), where $u_{1 \pm}^{(f t)}$ are defined by (4.19) and (4.20) with $E_{1 r}^{t}$ replacing $E_{d}^{t}$ and where the orthogonality of $u_{1-}^{(f t)}$ and $u_{1-}^{(f t)}$ follows from the remark after (4.25). Now, from (17.1), we have

$$
u_{1-}^{(f t)}(\omega)=p_{-}^{(f t)}(\omega)
$$


The function $p_{-}^{(f t)}$, defined by 4.11) and (4.12), is a given quantity. Clearly, the condition that minimizes $M(t)$ is

$$
u_{1+}^{(f t)}(\omega)=p_{1+}^{(f t)}(\omega)=\frac{1}{2 \pi i} \int_{-\infty}^{\infty} \frac{H_{-}^{f}\left(\omega^{\prime}\right) E_{1 F}^{t}\left(\omega^{\prime}\right)}{\omega^{\prime}-\omega^{-}} d \omega^{\prime}=0
$$

where (4.21) 2 has been used. It follows from (7.3) that

$$
E_{d m}^{t}(\omega)=\frac{p_{+}^{(f t)}(\omega)}{H_{-}^{f}(\omega)}=\frac{1}{2 \pi i H_{-}^{f}(\omega)} \int_{-\infty}^{\infty} \frac{H_{-}^{f}\left(\omega^{\prime}\right) E_{r+}^{t}\left(\omega^{\prime}\right)}{\omega^{\prime}-\omega^{-}} d \omega^{\prime}
$$

which is the optimal choice of $E_{d F}^{t}$. Let $E_{f}^{t}$ be the corresponding optimal choice of $E_{r 1}^{t}$, given by

$$
E_{f}^{t}(\omega)=E_{r+}^{t}(\omega)+E_{d m}^{t}(\omega)=\frac{p_{-}^{(f t)}(\omega)}{H_{-}^{f}(\omega)}=\frac{1}{2 \pi i H_{-}^{f}(\omega)} \int_{-\infty}^{\infty} \frac{H_{-}^{f}\left(\omega^{\prime}\right) E_{r+}^{t}\left(\omega^{\prime}\right)}{\omega^{\prime}-\omega^{+}} d \omega^{\prime}
$$

on using (4.11), and

$$
\left\|E_{f}^{t}\right\|^{2}=\frac{1}{2 \pi} \int_{-\infty}^{\infty} H\left(\omega^{\prime}\right)\left|E_{f}^{t}\left(\omega^{\prime}\right)\right|^{2} d \omega^{\prime} .
$$

The resulting free energy is given by (7.2) or, in the notation of (7.6),

$$
\begin{aligned}
\psi_{f}(t) & =\phi(t)+\left\|E_{f}^{t}\right\|^{2} \\
& =\phi(t)+\left\|p_{-}^{(f t)}\right\|_{L}^{2} .
\end{aligned}
$$

We write

$$
E_{f}^{t}(\omega)=E_{f+}^{t}(\omega)+E_{f-}^{t}(\omega)
$$

where $E_{f+}^{t}$ is analytic in $\Omega^{-}$and corresponds to a history before time $t$, while $E_{f-}^{t}$ is analytic in $\Omega^{+}$and corresponds to a continuation after time $t$. Formal expressions can be given for these two quantities by using the device introduced in (4.11) and (4.12). If the zeros in $H_{-}^{f}$ are not permuted $(f=1)$, then $E_{f+}^{t}$ vanishes. This case is readily identified as the minimum free energy derived in [14] and given by (2.36), with $E_{f}^{t}$ identified as the negative of the optimal continuation, as stated in (4.17).

If all the zeros are permuted $(f=N)$, then $E_{f-}^{t}$ vanishes. To see this, we observe that if the contour is completed on $\Omega^{(-)}$, the integral in (7.5) picks out the singularities due to $H_{-}^{f}$. These are then cancelled by the singularities of $H_{-}^{f}$ in the denominator so that the final singularities of $E_{f}^{t}$ are at the zeros of $H_{-}^{f}$. The case where $E_{f-}^{t}$ vanishes, corresponding to the maximum free energy, has $E_{f}^{t}$ as an optimal history up to time $t$. This is the solution given by (5.7) with associated free energy given by (5.6). We note (4.18) and (5.5) in this context.

If some zeros are permuted, we have an intermediate situation where neither $E_{f+}^{t}$ nor $E_{f-}^{t}$ vanish.

Just as in (2.40), we can write $\psi_{f}(t)$ in the form

$$
\begin{gathered}
\psi(t)=\phi(t)+\frac{i}{4 \pi^{2}} \int_{-\infty}^{\infty} \frac{\bar{E}_{r+}^{t}\left(\omega_{1}\right) L_{f}\left(\omega_{1}, \omega_{2}\right) E_{r+}^{t}\left(\omega_{2}\right)}{\omega_{1}^{+}-\omega_{2}^{-}} d \omega_{1} d \omega_{2}, \\
L_{f}\left(\omega_{1}, \omega_{2}\right)=H_{+}^{f}\left(\omega_{1}\right) H_{-}^{f}\left(\omega_{2}\right) .
\end{gathered}
$$


It is clear that properties P1-P3 of a free energy follow from (7.7); see (2.38) and related discussion, noting also (4.24). All that remains is to show that

$$
T(t) \dot{E}(t)=\dot{W}(t)=\dot{\psi}_{f}(t)+D_{f}(t), \quad D_{f}(t) \geq 0 .
$$

Using (4.11), we can write, with the help of (7.7),

$$
\begin{aligned}
W(t) & =\phi(t)+\left\|p_{-}^{(f t)}-p_{+}^{(f t)}\right\|_{L}^{2} \\
& =\phi(t)+\left\|p_{-}^{(f t)}\right\|_{L}^{2}+\left\|p_{+}^{(f t)}\right\|_{L}^{2} \\
& =\psi_{f}(t)+\left\|p_{+}^{(f t)}\right\|_{L}^{2}
\end{aligned}
$$

where the orthogonality of $p_{-}^{(f t)}, p_{+}^{(f t)}$ follows from the remark after (4.25). Comparing (7.10) and (7.11) $)_{3}$, we see that

$$
\begin{aligned}
& D_{f}(t)=\frac{d}{d t}\left\|p_{+}^{(f t)}\right\|_{L}^{2}=\left|K_{f}(t)\right|^{2}, \\
& K_{f}(t)=\left\langle H_{+}^{(f)}, E_{r+}^{t}\right\rangle .
\end{aligned}
$$

We have used 4.13 1 , 4.15) and their complex conjugates, together with 4.13) 3 , in writing (7.12). The quantity $D_{f}$ is clearly non-negative. It can also be written as (cf. (2.41) )

$$
D_{f}(t)=\frac{1}{4 \pi^{2}} \int_{-\infty}^{\infty} \bar{E}_{r+}^{t}\left(\omega_{1}\right) L_{f}\left(\omega_{1}, \omega_{2}\right) E_{r+}^{t}\left(\omega_{2}\right) d \omega_{1} d \omega_{2}
$$

in the notation of (7.9).

Let $E_{f o \pm}^{t}(s), s \in \mathbb{R}$, be the time domain history and continuation, corresponding to $E_{f \pm}^{t}(\omega)$. It can be shown [17] that the quantities $E_{f o \pm}^{t}( \pm \infty)$ (associated with derived, as opposed to given histories/continuations) are generally non-zero and that $E_{f o+}^{t}\left(0^{+}\right) \neq$ $E_{f o-}^{t}\left(0^{-}\right)$.

Partial orderings among the $\psi_{f}$ were established in [17] for the case of simple poles and zeros on the imaginary axis. This can be generalized in the following way. Starting from $\psi_{m}$, one can show that interchanging one zero at a time, the resulting $\psi_{f}$ is not less than the previous one. This leads to various chains of inequalities up to the maximum free energy $\psi_{M}$, each chain corresponding to a particular order of choosing the interchanged zeros. The quantity

$$
I_{l}\left(\omega_{1}, \omega_{2}\right)=\frac{X_{l}\left(\omega_{1}\right)}{X_{l}\left(\omega_{2}\right)}
$$

with $X_{l}$ defined by (4.9) is the interchange operator, removing a factor $\left(\omega_{2}-\bar{\eta}_{l}\right)$ from $H_{-}^{f}\left(\omega_{2}\right)$ and $\left(\omega_{1}-\eta_{l}\right)$ from $H_{+}^{f}\left(\omega_{1}\right)$ while attaching factors $\left(\omega_{2}-\eta_{l}\right),\left(\omega_{1}-\bar{\eta}_{l}\right)$ to $H_{-}^{f}\left(\omega_{2}\right)$, $H_{+}^{f}\left(\omega_{1}\right)$, respectively. Then, using (7.9), we need to show that

$$
\frac{i}{4 \pi^{2}} \int_{-\infty}^{\infty} \frac{\bar{E}_{r+}^{t}\left(\omega_{1}\right) H_{+}^{f}\left(\omega_{1}\right)\left[I_{l}\left(\omega_{1}, \omega_{2}\right)-1\right] H_{-}^{f}\left(\omega_{2}\right) E_{r+}^{t}\left(\omega_{2}\right)}{\omega_{1}^{+}-\omega_{2}^{-}} d \omega_{1} d \omega_{2} \geq 0 .
$$

This follows from the relation

$$
I_{l}\left(\omega_{1}, \omega_{2}\right)-1=-i \frac{\left(I m \eta_{l}\right)\left(\omega_{1}-\omega_{2}\right)}{\left(\omega_{1}-\eta_{l}\right)\left(\omega_{2}-\bar{\eta}_{l}\right)}
$$

since $I m \eta_{l}>0$ for all $l$. 
8. Explicit forms. Expressions for $p_{ \pm}^{(f t)}$ and $K_{f}$ are now derived, in terms of the parameters of the isolated singularities, which allow straightforward evaluation of $\psi_{f}$ and $D_{f}$ through (7.7) and (7.12). The developments generalize those described in [14, 17, 20], though the results are of necessity not so explicit as in those references.

The quantities $p_{ \pm}^{(f t)}(\omega)$, defined by (4.11) and (4.12), have the form

$$
\begin{aligned}
p_{-}^{(f t)}(\omega) & =\left.\sum_{l=1}^{S} Z_{l}\left(\omega, \omega^{\prime}, \bar{\zeta}_{l}\right)\right|_{\omega^{\prime}=\bar{\zeta}_{l}} \\
Z_{l}\left(\omega, \omega^{\prime}, \bar{\zeta}_{l}\right) & =\frac{1}{\left(m_{l}-1\right) !}\left(\frac{\partial}{\partial \omega^{\prime}}\right)^{m_{l}-1}\left[\frac{H_{-}^{f}\left(\omega^{\prime}\right)\left(\omega^{\prime}-\bar{\zeta}_{l}\right)^{m_{l}} E_{r+}^{t}\left(\omega^{\prime}\right)}{\omega-\omega^{\prime}}\right], \\
p_{+}^{(f t)}(\omega) & =p_{-}^{(f t)}(\omega)-H_{-}^{f}(\omega) E_{+}^{t}(\omega)
\end{aligned}
$$

as may be seen by closing the contour on $\Omega^{(-)}$. Observe that $p_{-}^{(f t)}(\omega)$ depends on $E_{r}^{t}$ only through the quantities $E_{r+}^{t}\left(\bar{\zeta}_{l}, j\right)$, defined by (6.1) so that it is a minimal state variable, by Proposition 6.1 and as must be the case by Corollary 4.2. Also, $E_{f}^{t}$ is given by (7.5). The decomposition (7.8) is given explicitly in [17, 20]. The corresponding expressions in the general case are very complicated but, as noted earlier, can be given formally by the device used in (4.11), (4.12).

The free energy $\psi_{f}(t)$, given by (7.7), has the form

$$
\psi(t)=\phi(t)+\left.i \sum_{n, l=1}^{S} \Delta_{n l}\left\{\frac{\left(\omega_{1}-\zeta_{n}\right)^{m_{n}}\left(\omega_{2}-\bar{\zeta}_{l}\right)^{m_{l}} A_{f}^{t}\left(\omega_{1}, \omega_{2}\right)}{\omega_{1}-\omega_{2}}\right\}\right|_{\substack{\omega_{1}=\zeta_{n} \\ \omega_{2}=\bar{\zeta}_{l}}}
$$

where, in terms of $L_{f}$, defined by $(7.9)_{2}$,

$$
A_{f}^{t}\left(\omega_{1}, \omega_{2}\right)=\bar{E}_{r+}^{t}\left(\omega_{1}\right) L_{f}\left(\omega_{1}, \omega_{2}\right) E_{r+}^{t}\left(\omega_{2}\right)
$$

and where $\Delta_{n l}$ is a differential operator given by

$$
\Delta_{n l}=\frac{1}{\left(m_{n}-1\right) !} \frac{1}{\left(m_{l}-1\right) !}\left(\frac{\partial}{\partial \omega_{1}}\right)^{m_{n}-1}\left(\frac{\partial}{\partial \omega_{2}}\right)^{m_{l}-1} .
$$

The quantity $K_{f}(t)$, given by $(7.12)_{2}$, is best evaluated using (4.14) and (8.1) to give

$$
K_{f}(t)=-\left.i \sum_{l=1}^{S} \frac{1}{\left(m_{l}-1\right) !}\left(\frac{\partial}{\partial \omega^{\prime}}\right)^{m_{l}-1}\left[H_{-}^{f}\left(\omega^{\prime}\right)\left(\omega^{\prime}-\bar{\zeta}_{l}\right)^{m_{l}} E_{r+}^{t}\left(\omega^{\prime}\right)\right]\right|_{\omega^{\prime}=\bar{\zeta}_{l}}
$$

and

$$
D_{f}(t)=\left|K_{f}(t)\right|^{2}=\left.\sum_{n, l=1}^{S} \Delta_{n l}\left\{\left(\omega_{1}-\zeta_{l}\right)^{m_{n}}\left(\omega_{2}-\bar{\zeta}_{l}\right)^{m_{l}} A_{f}^{t}\left(\omega_{1}, \omega_{2}\right)\right\}\right|_{\substack{\omega_{1}=\zeta_{n} \\ \omega_{2}=\bar{\zeta}_{l}}} .
$$

The fact that $K_{f}$ is a linear function of the quantity $E_{r+}^{t}\left(\bar{\zeta}_{l}, j\right)$ and therefore of the vector elements $\bar{e}_{l j}$, defined by $(\underline{6.4})_{3}$, means that $D_{f}$, given by $(\mathbf{7 . 1 2})$, will be a quadratic form as in (6.7), where $\boldsymbol{\Gamma}$ is positive semi-definite rather than strictly positive definite.

The $N$ free energies defined by different factorizations are all in a convex set which we denote by $\mathcal{F}$. 
From Section [6 we see that the boundary of the convex set $\mathcal{F}$ will be indicated by the breakdown in the positive definiteness of either $\mathbf{C}$ or $\boldsymbol{\Gamma}$ or both. Thus we are on the boundary of $\mathcal{F}$ in the sense that the positive definiteness of $\boldsymbol{\Gamma}^{f}$ is breaking down to positive semi-definiteness. In this sense, the free energies formed from the different factorizations are extrema, which is consistent with the derivation of (7.7).

9. Proposed form of the physical free energy and dissipation. In this section, we consider a physical hypothesis and other assumptions that lead to a prediction for the form of the physical free energy and its associated total dissipation and rate of dissipation, using the family of free energies $\mathcal{F}$ derived in Section 7 .

The convexity of $\mathcal{F}$ means that we can form a family of free energies given by

$$
\psi(t)=\sum_{f} \lambda_{f} \psi_{f}(t), \quad \sum_{f} \lambda_{f}=1, \quad \lambda_{f} \geq 0, \quad f=1,2, \ldots, N,
$$

where the sum is in general over all $N$ factorizations. Clearly $\psi_{m}(t) \leq \psi(t) \leq \psi_{M}(t)$.

REMARK 9.1. Observe that a given free energy that is a function of the minimal state is characterized by the number $X$ of non-zero independent elements in the matrix $\mathbf{C}$ in (6.5), which is given by

$$
X=\frac{q}{2}(q+1)
$$

where $q$ is defined by (4.5). Comparing this with the number of $\lambda_{f}$ in (9.1), namely $N=2^{p}$ defined by (4.7), we see that $N=X$ for $p=0, N<X$ for $p=1,2,3$ and $N>X$ for $p>3$. In fact, it becomes much larger, as $p$ increases. Each non-zero, independent element of $\mathbf{C}$ is a linear function of the $\lambda_{f}$ since the matrix $\mathbf{C}_{f}$ corresponding to $\psi_{f}$ is a given quantity. Thus, for $p>3$, many different choices of $\lambda_{f}$ will correspond to the same free energy.

For $p=1,2,3$, the form (9.1) will not represent all possible free energies that are functions of the minimal state.

Using (7.9), we can write

$$
\begin{gathered}
\psi(t)=\phi(t)+\frac{i}{4 \pi^{2}} \int_{-\infty}^{\infty} \frac{\bar{E}_{r+}^{t}\left(\omega_{1}\right) L\left(\omega_{1}, \omega_{2}\right) E_{r+}^{t}\left(\omega_{2}\right)}{\omega_{1}^{+}-\omega_{2}^{-}} d \omega_{1} d \omega_{2}, \\
L\left(\omega_{1}, \omega_{2}\right)=\sum_{f} \lambda_{f} H_{+}^{f}\left(\omega_{1}\right) H_{-}^{f}\left(\omega_{2}\right) .
\end{gathered}
$$

The essential difficulty in identifying the physical free energy, which we denote by $\psi_{p}$, is that of finding a criterion that would distinguish it from other free energies.

REMARK 9.2. The first and basic assumption will be that the physical free energy is given by the form (9.2) for a particular choice of the coefficients $\lambda_{f}$.

Our approach is to determine values of the coefficients $\lambda_{f}, f=1,2, \ldots, N$, which yield the physical free energy, on the basis of a physical hypothesis which will now be discussed.

We will focus on the symmetries of $H$ under interchanges of parameters and we will refer to these as parametric symmetries. It must be emphasized that when discussing parametric symmetries, we must be clear on what is being adopted as the set of independent parameters. These are generally taken to be those in (3.1)-(3.3). However, in the 
present context, and perhaps in general, the natural choice of independent parameters of the material is the zeros and singular points of $H$, their respectives orders and $H_{\infty}$. The zeros and singular points occur in complex conjugate pairs. One of these, say the one in the upper half plane, will be chosen because the real parts are not arbitrary: either they are zero or equal in pairs, as discussed in Sections 3 and 4. Thus, the material is characterized by $\left(n_{l}, \eta_{l}\right), l=2,3, \ldots, Z,\left(m_{l}, \zeta_{l}\right), l=1,2, \ldots, S$ and $H_{\infty}$. We will refer to these as the standard parameters.

It may be observed from (4.4) 4 that $H$ is symmetric under the following interchanges:

(1) each pair $\eta_{l}, \bar{\eta}_{l}, l=1,2, \ldots, Z$;

(2) each pair $\left(n_{i}, \eta_{i}\right)$ and $\left(n_{j}, \eta_{j}\right), i, j=1,2, \ldots, Z$;

(3) each pair $\left(n_{i}, \bar{\eta}_{i}\right)$ and $\left(n_{j} \bar{\eta}_{j}\right), i, j=1,2, \ldots, Z$;

(4) each pair $\zeta_{l}, \bar{\zeta}_{l}, l=1,2, \ldots, S$;

(5) each pair $\left(m_{i}, \zeta_{i}\right)$ and $\left(m_{j}, \zeta_{j}\right), i, j=1,2, \ldots, S$;

(6) each pair $\left(m_{i}, \bar{\zeta}_{i}\right)$ and $\left(m_{j}, \bar{\zeta}_{j}\right), i, j=1,2, \ldots, S$.

Symmetries (21), (3) and (5), (6) include the symmetry due to (4.6) which is a consequence of the reality constraint (2.13): if a pair of zeros related by (4.6) are interchanged, then $H$ is unchanged. We will not consider this symmetry separately. It does not apply to the individual $\psi_{f}$ but is present in our final result.

The symmetries given above are a consequence of (a) the form of $G_{F}^{\prime}$ as a sum of pole terms of the form (3.1), or $G^{\prime}$ as given by (3.2); and (b) the symmetric singularity structure resulting from (2.14).

Since 30

$$
G_{F}^{\prime}(\omega)=-\frac{1}{\pi} \int_{-\infty}^{\infty} \frac{H\left(\omega^{\prime}\right)}{\omega^{\prime}\left(\omega^{\prime}-\omega^{-}\right)} d \omega^{\prime},
$$

the symmetries of $H$ are shared by $G_{F}^{\prime}$ and indeed $G^{\prime}$ on taking an inverse Fourier transform. Similarly, relation (2.23), with (2.14), imply that the stress function $T$ possesses the same symmetries.

All the listed symmetries are an intrinsic property of the work function given by (2.27), in part because of its single frequency structure. On the other hand, they are not the property of the individual free energies in (9.1). Using the detailed forms given by (17.9) and (4.8), we see that the individual $\psi_{f}$, and therefore any sum of these, possess symmetries (5) and (6). The minimum and maximum free energies also have symmetries (21) and (3).

Symmetry (4) is definitely excluded for all $\psi_{f}$ in that such an exchange interferes with the defining analytic structure of each $\psi_{f}$. In fact, if such an exchange were made and the relevant $H_{ \pm}$were expressed as sums of poles by the use of the partial fractions theorem, the exchanged poles would give a vanishing contribution, on application of Cauchy's theorem. Thus, this symmetry cannot in general be a property of a free energy given by the form (9.2). This is connected with the two frequency, as opposed to one frequency, structure of the integrals defining $\psi_{f}$.

REMARK 9.3. Let us adopt the point of view that physical energy measures possess a high degree of parametric symmetry, including all or nearly all of those listed above. The individual $\psi_{f}$ are approximations to the physical free energy and we suggest that this approximate status is correlated with their low level of parametric symmetry. 
Carrying this line of argument further, we formulate the following:

Hypothesis 9.1. The physical free energy $\psi_{p}$ is the choice of free energy of form (9.2) with the closest possible level of parametric symmetry to that of the work function, allowed by the structure of (9.2).

This is referred to as the Hypothesis of Maximum Parametric Symmetry.

Since, from (7.10)

$$
\psi_{f}(t)+\mathcal{D}_{f}(t)=W(t), \quad \mathcal{D}_{f}(t)=\int_{-\infty}^{t} D_{f}(s) d s,
$$

the hypothesis tells us that the total dissipation $\mathcal{D}_{f}$ and the rate of dissipation $D_{f}$ will have the same level of parametric symmetry as $\psi_{f}$; also the physical dissipation $\mathcal{D}_{p}$ and the physical rate of dissipation $D_{p}$ will have the same level of parametric symmetry as $\psi_{p}$.

The quantity $\psi_{p}$ will have, by construction, symmetries (5) and (6) and cannot have symmetry (4), nor can it have symmetries (2) and (3) separately because in general they can give rise to situations where, in effect, $a_{l}^{f}+b_{l}^{f} \neq n_{l}$. However, we can enforce a weaker symmetry, namely that under the interchange of $\left(n_{i}, \eta_{i}, \bar{\eta}_{i}\right)$ and $\left(n_{j}, \eta_{j}, \bar{\eta}_{j}\right)$ for each $i, j=1,2 \ldots, Z$. Thus, we will require the following symmetries as the largest set compatible with the structure of (9.2): symmetry under the interchange of

(1) each pair $\eta_{l}, \bar{\eta}_{l}, l=1,2, \ldots, Z$;

(5) each pair $\left(m_{i}, \zeta_{i}\right)$ and $\left(m_{j}, \zeta_{j}\right), i, j=1,2, \ldots, S$;

(6) each pair $\left(m_{i}, \bar{\zeta}_{i}\right)$ and $\left(m_{j}, \bar{\zeta}_{j}\right), i, j=1,2, \ldots, S$.

(7) each pair $\left(n_{i}, \eta_{i}, \bar{\eta}_{i}\right)$ and $\left(n_{j}, \eta_{j}, \bar{\eta}_{j}\right), i, j=1,2, \ldots, Z$;

By restricting the summation in (9.1) 1 to include only the minimum and maximum free energies, we could maintain symmetries (51) and (61) but we would lose symmetry (1) - except where all $\eta_{l}, \bar{\eta}_{l}, l=1,2, \ldots, Z$, are interchanged. In any case, such an expression could be objected to on general grounds in that in a situation where there are many approximations $\psi_{f}$ to $\psi_{p}$, only two of these are used to estimate $\psi_{p}$, or put differently, the available data is under-utilized.

Later, it will be rendered more sharply clear that symmetries (1) and (7) are the largest set of symmetries achievable among the zeros $\eta_{l}$, at least under Hypothesis 9.2 below.

Since symmetries (5) and (6) are present in any choice of $\psi_{f}$, we need to enforce only symmetries (1) and (7). This will be done in two ways. The first is a direct proof and involves certain weak and natural assumptions. The second is indirect, simpler and involves fewer assumptions.

We will generally omit reference to the zeros in $H_{ \pm}$at the origin in the following argument, because they trivially fulfil all requirements.

If $\psi=\psi_{p}$, the kernel

$$
L_{p}\left(\omega_{1}, \omega_{2}\right)=\sum_{f} \lambda_{f}^{(p)} H_{+}^{f}\left(\omega_{1}\right) H_{-}^{f}\left(\omega_{2}\right)
$$

must have the required level of symmetry. 
We can write in general

$$
\begin{aligned}
\lambda_{f}^{(p)} & =\chi_{f}\left(\eta_{l}, \bar{\eta}_{l}, a_{l}^{f}, b_{l}^{f}, l=2,3, \ldots, Z\right) \\
& =\mu_{f}\left(\eta_{l}, \bar{\eta}_{l}, a_{l}^{f}, n_{l}, l=2,3, \ldots, Z\right)
\end{aligned}
$$

where $a_{l}^{f}$ and $b_{l}^{f}=n_{l}-a_{l}^{f}$ are introduced in (4.8). Each $H_{+}^{f}\left(\omega_{1}\right) H_{-}^{f}\left(\omega_{2}\right)$ and therefore each $\psi_{f}$ is symmetric under any interchange $\left(\eta_{i}, \bar{\eta}_{i}, a_{i}^{f}, n_{i}\right) \leftrightarrow\left(\eta_{j}, \bar{\eta}_{j}, a_{j}^{f}, n_{j}\right)$ for $i, j=$ $2,3, \ldots, Z$. This symmetry will be assumed for $\psi_{p}$, since it is a property of each estimate of this quantity, so it must apply to each $\mu_{f}$. The following is now introduced.

Hypothesis 9.2. The coefficients $\lambda_{f}^{(p)}$ are assumed to possess symmetries (1) and (7).

This might not seem to be a particularly natural assumption. A possibly more natural assumption would be that the coefficients are independent of the positions of the zeros $\eta_{l}, \bar{\eta}_{l}$. However, it turns out that this is a consequence of Hypothesis 9.2

The subscript $f$ will be dropped from $\chi_{f}$ and $\mu_{f}$. In other words, the same function applies in each case; there will be no extra parameters, for example multiplying constants, introducing a further variability between the $\lambda_{f}^{(p)}$. It will be unnecessary to make this assumption in the second, indirect proof presented below.

Symmetry (7), together with the symmetry, specified above, under the exchange of all four parameters, yields that $\mu_{f}\left(\eta_{l}, \bar{\eta}_{l}, a_{l}^{f}, n_{l}, l=2,3, \ldots, Z\right)$ is symmetric under the interchange $a_{i}^{f} \leftrightarrow a_{j}^{f}$. There is the problem that the resulting factors will be unallowable if $n_{i}<a_{j}^{f}$ or $n_{j}<a_{i}^{f}$. However, let us impose the property that

$$
\mu\left(\eta_{l}, \bar{\eta}_{l}, a_{l}^{f}, n_{l}, l=2,3, \ldots, Z\right)=0
$$

if any $n_{i}<a_{i}^{f}$ and that $\mu$ is symmetric under the interchange of any $a_{i}^{f}, a_{j}^{f}, i, j=$ $2,3, \ldots, Z$.

The exchange of a given $\eta_{l}, \bar{\eta}_{l}$ in a particular $H_{+}^{f}\left(\omega_{1}\right) H_{-}^{f}\left(\omega_{2}\right)$ yields $H_{+}^{f^{\prime}}\left(\omega_{1}\right) H_{-}^{f^{\prime}}\left(\omega_{2}\right)$ where $f^{\prime}$ denotes the factorization with $a_{l}^{f}, b_{l}^{f}$ interchanged. Thus, since $\chi, \mu$ possess symmetry (1), the symmetry will prevail provided that

$$
\begin{aligned}
& \chi\left(\eta_{l}, \bar{\eta}_{l}, a_{l}^{f}, b_{l}^{f}, l=2,3, \ldots, Z\right)=\chi\left(\eta_{l}, \bar{\eta}_{l}, b_{l}^{f}, a_{l}^{f}, l=2,3, \ldots, Z\right), \\
& \mu\left(\eta_{l}, \bar{\eta}_{l}, a_{l}^{f}, n_{l}, l=2,3, \ldots, Z\right)=\mu\left(\eta_{l}, \bar{\eta}_{l}, b_{l}^{f}, n_{l}, l=2,3, \ldots, Z\right) .
\end{aligned}
$$

Under the interchange $\left(n_{i}, \eta_{i}, \bar{\eta}_{i}\right) \leftrightarrow\left(n_{j}, \eta_{j}, \bar{\eta}_{j}\right)$, the factors

$$
\left(\omega_{1}-\eta_{i}\right)^{a_{i}^{f}}\left(\omega_{1}-\bar{\eta}_{i}\right)^{n_{i}-a_{i}^{f}}, \quad\left(\omega_{1}-\eta_{j}\right)^{a_{j}^{f}}\left(\omega_{1}-\bar{\eta}_{j}\right)^{n_{j}-a_{j}^{f}}
$$

in $H_{+}^{f}\left(\omega_{1}\right)$ become

$$
\left(\omega_{1}-\eta_{j}\right)^{a_{i}^{f}}\left(\omega_{1}-\bar{\eta}_{j}\right)^{n_{j}-a_{i}^{f}}, \quad\left(\omega_{1}-\eta_{i}\right)^{a_{j}^{f}}\left(\omega_{1}-\bar{\eta}_{i}\right)^{n_{i}-a_{j}^{f}} .
$$

This is equivalent to the interchange $a_{i}^{f} \leftrightarrow a_{j}^{f}$. A similar argument applies to $H_{-}^{f}\left(\omega_{2}\right)$. Let

$$
n_{m}=\max \left\{n_{1}, n_{2}, \ldots, n_{Z}\right\}
$$

and

$$
L_{p}\left(\omega_{1}, \omega_{2}\right)=\sum^{(e)} \lambda_{f}^{(p)} H_{+}^{f}\left(\omega_{1}\right) H_{-}^{f}\left(\omega_{2}\right)
$$


where the notation $\sum^{(e)}$ indicates an expanded summation covering all $a_{l}^{f} \leq n_{m}$ for each $l$. Various terms, those that are not allowed, will vanish by virtue of (9.5). However, as an algebraic expression, $L_{p}$ has the required symmetry under the interchange of any pair $a_{i}^{f}, a_{j}^{f}$.

We can impose property (9.6) and the symmetry under the exchange of $a_{i}^{f}, a_{j}^{f}, i, j=$ $2,3, \ldots, Z$ in the simplest way by taking

$$
\chi\left(\eta_{l}, \bar{\eta}_{l}, a_{l}^{f}, b_{l}^{f}, l=2,3, \ldots, Z\right)=\prod_{l=2}^{Z} f\left(b_{l}^{f} ; n_{l}, \eta_{l}, \bar{\eta}_{l}\right) f\left(a_{l}^{f} ; n_{l}, \eta_{l}, \bar{\eta}_{l}\right)
$$

where $f\left(z ; n_{l}, \eta_{l}, \bar{\eta}_{l}\right)$ is a function with the property that $f\left(-n ; n_{l}, \eta_{l}, \bar{\eta}_{l}\right)$ vanishes for $n=1,2, \ldots$. We will make the assumption that $f$ is an analytic function of the first argument with no singularities and no other zeros on the finite complex plane. The justification for this is that the physical significance of any such singularities or zeros would by unclear. Then 38] $f$ is uniquely given by

$$
f\left(z ; n_{l}, \eta_{l}, \bar{\eta}_{l}\right)=\frac{Y\left(n_{l}, \eta_{l}, \bar{\eta}_{l}\right)}{\Gamma(z+1)}
$$

in terms of the standard Gamma function, where $Y$ is an unspecified function of $n_{l}, \eta_{l}, \bar{\eta}_{l}$. Thus, we put

$$
\begin{aligned}
\mu\left(\eta_{l}, \bar{\eta}_{l}, a_{l}^{f}, n_{l}, l=2,3, \ldots, Z\right) & =\chi\left(\eta_{l}, \bar{\eta}_{l}, a_{l}^{f}, b_{l}^{f}, l=2,3, \ldots, Z\right) \\
& =B \prod_{l=2}^{Z} \frac{Y^{2}\left(n_{l}, \eta_{l}, \bar{\eta}_{l}\right)}{\Gamma\left(n_{l}-a_{l}^{f}+1\right) \Gamma\left(a_{l}^{f}+1\right)} .
\end{aligned}
$$

The quantity $B$ is introduced to ensure that $(9.1)_{2}$ holds. We put

$$
Y^{2}\left(n_{l}, \eta_{l}, \bar{\eta}_{l}\right)=A_{l}\left(n_{l}, \eta_{l}, \bar{\eta}_{l}\right) \Gamma\left(n_{l}+1\right)
$$

and we absorb $\prod_{l=2}^{l} A_{l}\left(n_{l}, \eta_{l}, \bar{\eta}_{l}\right)$ into the factor $B$, so that

$$
\chi\left(\eta_{l}, \bar{\eta}_{l}, a_{l}^{f}, b_{l}^{f}, l=2,3, \ldots, Z\right)=B \prod_{l=2}^{Z}{ }^{n_{l}} \mathbf{C}_{a_{l}^{f}}
$$

in terms of the standard notation defined by 6.2 . The quantity $B$ can be evaluated by applying the constraint (9.1) ${ }_{2}$. As $f$ varies from 1 to $N$, each $a_{l}^{f}$ will independently take all integer values in the range $\left[0, m_{l}\right]$, so that

$$
\sum_{f=1}^{N} \lambda_{f}^{(p)}=B \sum_{a_{1}=0}^{m_{1}} \cdots \sum_{a_{Z}=0}^{m_{Z}} \prod_{l=2}^{Z}{ }^{n_{l}} \mathbf{C}_{a_{l}^{f}}
$$

and

$$
B=\frac{1}{N}=2^{-p}
$$

where $p$ is given by (4.7). We conclude that the coefficients $\lambda_{f}^{(p)}$ do not depend on $\eta_{l}, \bar{\eta}_{l}$. The quantities ${ }^{n_{l}} \mathbf{C}_{a_{l}^{f}}$ are of course the binomial coefficients and there is the immediate 
possibility that (9.1) 1 can be condensed into a closed form. This is in fact the case: one obtains

$$
\begin{aligned}
& L_{p}\left(\omega_{1}, \omega_{2}\right) \\
& =B H_{\infty} \omega_{1} \omega_{2} \frac{\sum_{a_{1}=0}^{m_{1}} \cdots \sum_{a_{Z}=0}^{m_{Z}} \prod_{l=2}^{Z}{ }^{n_{l}} \mathbf{C}_{a_{l}^{f}}\left[\left(\omega_{1}-\eta_{l}\right)\left(\omega_{2}-\bar{\eta}_{l}\right)\right]^{a_{l}^{f}}\left[\left(\omega_{2}-\eta_{l}\right)\left(\omega_{1}-\bar{\eta}_{l}\right)\right]^{b_{l}^{f}}}{\prod_{l=1}^{S}\left[\left(\omega_{1}-\zeta_{l}\right)\left(\omega_{2}-\bar{\zeta}_{l}\right)\right]^{m_{l}}} \\
& =B H_{\infty} \omega_{1} \omega_{2} \frac{\prod_{l=2}^{Z}\left\{\left(\omega_{1}-\eta_{l}\right)\left(\omega_{2}-\bar{\eta}_{l}\right)+\left(\omega_{2}-\eta_{l}\right)\left(\omega_{1}-\bar{\eta}_{l}\right)\right\}^{n_{l}}}{\prod_{l=1}^{S}\left[\left(\omega_{1}-\zeta_{l}\right)\left(\omega_{2}-\bar{\zeta}_{l}\right)\right]^{m_{l}}} \\
& =B H_{+}\left(\omega_{1}\right) \prod_{l=2}^{Z}\left\{1+I_{l}\left(\omega_{1}, \omega_{2}\right)\right\}^{n_{l}} H_{-}\left(\omega_{2}\right) .
\end{aligned}
$$

The quantity $I_{l}$ is the interchange operator for the zeros $\eta_{l}, \bar{\eta}_{l}$, defined by (7.14). We can also write $L_{p}$ in the form

$$
L_{p}\left(\omega_{1}, \omega_{2}\right)=H_{\infty} \omega_{1} \omega_{2} \frac{\prod_{l=2}^{Z}\left\{\omega_{1} \omega_{2}+\left|\eta_{l}\right|^{2}-\left(\omega_{1}+\omega_{2}\right) R e \eta_{l}\right\}^{n_{l}}}{\prod_{l=1}^{S}\left[\left(\omega_{1}-\zeta_{l}\right)\left(\omega_{2}-\bar{\zeta}_{l}\right)\right]^{m_{l}}} .
$$

The kernel $L_{p}$ has the required symmetries. It also obeys the reality constraint of symmetry between zeros related by (4.6), because for each factor

$$
\left\{\omega_{1} \omega_{2}+\left|\eta_{l}\right|^{2}-\left(\omega_{1}+\omega_{2}\right) R e \eta_{l}\right\}^{n_{l}}
$$

where $\eta_{l}$ is off the imaginary axis, there will be another factor

$$
\left\{\omega_{1} \omega_{2}+\left|\eta_{l}\right|^{2}+\left(\omega_{1}+\omega_{2}\right) R e \eta_{l}\right\}^{n_{l}}
$$

and the product is unchanged by a change of sign of $R e \eta_{l}$.

It will be observed that, except in the case where $n_{l}=1, l=2,3, \ldots, Z$, the minimum and maximum free energies have least weight of all the $\psi_{f}$ in their contribution to $\psi_{p}$. This is reasonable in that those furthest from $\psi_{p}$ in magnitude contribute least. For the case of simple zeros, all $\psi_{f}$ have equal weight.

An alternative and simpler derivation of (9.8) is now presented. Hypothesis 9.2 is assumed. Choose the zeros initially to be all different, which is trivially possible if we use standard parameters as defined after Remark 9.2. In this case, symmetry (7) is symmetry under the interchange of $\left(\eta_{i}, \bar{\eta}_{i}\right)$ and $\left(\eta_{j}, \bar{\eta}_{j}\right), i, j=2,3, \ldots, Z$. Let us write $L_{p}$ in the form

$$
\begin{aligned}
L_{p}\left(\omega_{1}, \omega_{2}\right) & =B H_{+}\left(\omega_{1}\right) I\left(\omega_{1}, \omega_{2}\right) H_{-}\left(\omega_{2}\right), \\
I\left(\omega_{1}, \omega_{2}\right) & =\sum_{n=0}^{Z-1} \lambda_{n} \sum \prod_{l}^{(n)} I_{l}\left(\omega_{1}, \omega_{2}\right),
\end{aligned}
$$

where the notation $\sum \prod_{l}^{(n)}$ indicates the sum of all possible products of order $n$ chosen from $I_{l}, l=2,3, \ldots, Z$, no factor occurring with a power greater than unity. The reason we can assign a single coefficient $\lambda_{n}$ to the products of order $n$ is by application of 
symmetry (7), which, under Hypothesis 9.2 amounts to symmetry under the interchange of the $I_{l}$. Application of symmetry (1) gives the conditions (remembering the factors $H_{ \pm}$)

$$
I_{k}\left(\omega_{1}, \omega_{2}\right) I\left(\omega_{1}, \omega_{2} ; I_{k} \rightarrow 1 / I_{k}\right)=I\left(\omega_{1}, \omega_{2}\right), \quad k=2,3, \ldots, Z .
$$

The second factor on the left is the expression $(9.10)_{2}$ with $I_{k}$ replaced by its algebraic inverse, wherever it occurs. This yields the result that all the $\lambda_{n}$ are equal and we obtain

$$
I\left(\omega_{1}, \omega_{2}\right)=\prod_{l=2}^{Z}\left\{1+I_{l}\left(\omega_{1}, \omega_{2}\right)\right\} .
$$

Allowing the zeros to coalesce in a specified manner, we obtain $(9.8)_{3}$.

Note that the assumption made in the second paragraph after Hypothesis 9.2 together with those contained in (9.7) and in the paragraph after that equation, are avoided in this proof.

We can now place the assumption of symmetry (7) on a firm footing as the strongest

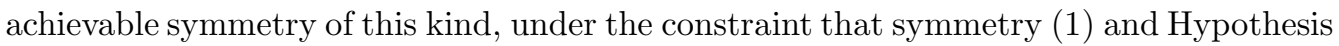
9.2 apply. Symmetries (2), (3) imply symmetry (7). If we assume that symmetries (2), (3) can be enforced on the form (9.4), a contradiction is obtained as follows: symmetries (1), (2) and (3) imply symmetries (11) and (7) which, in turn, imply the form (9.8). However, manifestly, (9.8) does not have symmetries (2), (3) individually.

From (9.2) and (9.8) or (9.9) we have that the physical free energy is given by

$$
\psi_{p}(t)=\phi(t)+\frac{i}{4 \pi^{2}} \int_{-\infty}^{\infty} \frac{\bar{E}_{r+}^{t}\left(\omega_{1}\right) L_{p}\left(\omega_{1}, \omega_{2}\right) E_{r+}^{t}\left(\omega_{2}\right)}{\omega_{1}^{+}-\omega_{2}^{-}} d \omega_{1} d \omega_{2} .
$$

The physical rate of dissipation is

$$
D_{p}(t)=\sum_{f} \lambda_{f}^{(p)}\left|K_{f}(t)\right|^{2}
$$

where $K_{f}$ is given by (7.12), which reduces to

$$
D_{p}(t)=\frac{1}{4 \pi^{2}} \int_{-\infty}^{\infty} \bar{E}_{r+}^{t}\left(\omega_{1}\right) L_{p}\left(\omega_{1}, \omega_{2}\right) E_{r+}^{t}\left(\omega_{2}\right) d \omega_{1} d \omega_{2}
$$

The quantities $\psi_{p}, D_{p}$ obey

$$
\dot{\psi}_{p}(t)+D_{p}(t)=T(t) \dot{E}(t)
$$

(cf. (7.10) ) which can be used also to deduce (9.12) from (9.11) with the aid of (2.22), (2.23) and contour integration. One can show that

$$
\int_{-\infty}^{\infty} \frac{\bar{E}_{r+}^{t}\left(\omega_{1}\right) L_{p}\left(\omega_{2}, \omega_{1}\right) E_{r+}^{t}\left(\omega_{2}\right)}{\omega_{1}^{+}-\omega_{2}^{-}} d \omega_{1} d \omega_{2}=0
$$

by completing the $\omega_{1}$ integration into a contour over $\Omega^{(+)}$. Thus, we can express $\psi_{p}(t)$ in an explicitly finite way [30]:

$$
\psi_{p}(t)=\phi(t)+\frac{i}{4 \pi^{2}} \int_{-\infty}^{\infty} \frac{\bar{E}_{r+}^{t}\left(\omega_{1}\right)\left[L_{p}\left(\omega_{1}, \omega_{2}\right)-L_{p}\left(\omega_{2}, \omega_{1}\right)\right] E_{r+}^{t}\left(\omega_{2}\right)}{\omega_{1}-\omega_{2}} d \omega_{1} d \omega_{2} .
$$


It is also possible to give more explicit expressions for $\psi_{p}(t), D_{p}(t)$ using the same approach as in (8.1), but applied directly to the double integration in (9.11). We obtain formulae similar to (8.2) and (8.4). Let

$$
A_{p}^{t}\left(\omega_{1}, \omega_{2}\right)=\bar{E}_{r+}^{t}\left(\omega_{1}\right) L_{p}\left(\omega_{1}, \omega_{2}\right) E_{r+}^{t}\left(\omega_{2}\right) .
$$

Then,

$$
\psi(t)=\phi(t)+\left.i \sum_{n, l=1}^{S} \Delta_{n l}\left\{\frac{\left(\omega_{1}-\zeta_{n}\right)^{m_{n}}\left(\omega_{2}-\bar{\zeta}_{l}\right)^{m_{l}} A_{p}^{t}\left(\omega_{1}, \omega_{2}\right)}{\omega_{1}-\omega_{2}}\right\}\right|_{\substack{\omega_{1}=\zeta_{n} \\ \omega_{2}=\bar{\zeta}_{l}}}
$$

where $\Delta_{n l}$ is the differential operator given by (8.3) and

$$
D_{p}(t)=\left.\sum_{n, l=1}^{S} \Delta_{n l}\left\{\left(\omega_{1}-\zeta_{n}\right)^{m_{n}}\left(\omega_{2}-\bar{\zeta}_{l}\right)^{m_{l}} A_{p}^{t}\left(\omega_{1}, \omega_{2}\right)\right\}\right|_{\substack{\omega_{1}=\zeta_{n} \\ \omega_{2}=\bar{\zeta}_{l}}} .
$$

Let us consider the special case of simple poles and zeros:

$$
m_{l}=1, \quad l=1,2, \ldots, S, \quad n_{l}=1, \quad l=2,3, \ldots, Z, \quad Z=S,
$$

where of course $n_{1}=1$ for all materials. Then

$$
\begin{aligned}
& \psi_{p}(t)=\phi(t)+\left.i \sum_{n, l=1}^{S}\left\{\frac{\left(\omega_{1}-\zeta_{n}\right)\left(\omega_{2}-\bar{\zeta}_{l}\right) A_{p}^{t}\left(\omega_{1}, \omega_{2}\right)}{\omega_{1}-\omega_{2}}\right\}\right|_{\substack{\omega_{1}=\zeta_{n} \\
\omega_{2}=\bar{\zeta}_{l}}} \\
& =\phi(t)+i H_{\infty} \sum_{n, l=1}^{S}\left\{\frac{\bar{E}_{r+}^{t}\left(\zeta_{n}\right) N_{p}\left(\zeta_{n}, \bar{\zeta}_{l}\right) E_{r+}^{t}\left(\bar{\zeta}_{l}\right)}{\zeta_{n}-\bar{\zeta}_{l}}\right\}, \\
& N_{p}\left(\zeta_{n}, \bar{\zeta}_{l}\right)=\frac{\zeta_{n} \bar{\zeta}_{l} \prod_{j=2}^{S}\left\{\zeta_{n} \bar{\zeta}_{l}+\left|\eta_{j}\right|^{2}-\left(\zeta_{n}+\bar{\zeta}_{l}\right) R e \eta_{j}\right\}}{\prod_{\substack{j=1 \\
j \neq n}}^{S}\left(\zeta_{n}-\zeta_{j}\right) \prod_{\substack{j=1 \\
j \neq l}}^{S}\left(\bar{\zeta}_{l}-\bar{\zeta}_{j}\right)} .
\end{aligned}
$$

Also,

$$
D_{p}(t)=H_{\infty} \sum_{n, l=1}^{S}\left\{\bar{E}_{r+}^{t}\left(\zeta_{n}\right) N_{p}\left(\zeta_{n}, \bar{\zeta}_{l}\right) E_{r+}^{t}\left(\bar{\zeta}_{l}\right)\right\} .
$$

We can write these formulae in the time domain as

$$
\begin{aligned}
\psi_{p}(t) & =\phi(t)+\frac{1}{2} \int_{0}^{\infty} d s_{1} \int_{0}^{\infty} d s_{2} E_{r}^{t}\left(s_{1}\right) F_{p}\left(s_{1}, s_{2}\right) E_{r}^{t}\left(s_{2}\right), \\
F_{p}\left(s_{1}, s_{2}\right) & =2 i H_{\infty} \sum_{n, l=1}^{S} \frac{N_{p}\left(\zeta_{n}, \bar{\zeta}_{l}\right)}{\zeta_{n}-\bar{\zeta}_{l}} e^{i\left(\zeta_{n} s_{1}-\bar{\zeta}_{l} s_{2}\right)},
\end{aligned}
$$

and

$$
\begin{aligned}
D_{p}(t) & =\frac{1}{2} \int_{0}^{\infty} d s_{1} \int_{0}^{\infty} d s_{2} E_{r}^{t}\left(s_{1}\right) M_{p}\left(s_{1}, s_{2}\right) E_{r}^{t}\left(s_{2}\right) \\
M_{p}\left(s_{1}, s_{2}\right) & =2 H_{\infty} \sum_{n, l=1}^{S} N_{p}\left(\zeta_{n}, \bar{\zeta}_{l}\right) e^{i\left(\zeta_{n} s_{1}-\bar{\zeta}_{l} s_{2}\right)}
\end{aligned}
$$


Let all the singularities be on the imaginary axis. The zeros will also have this property [14]. We put

$$
\begin{gathered}
\zeta_{n}=i \alpha_{n}, \quad n=1,2 \ldots, S, \quad \eta_{l}=i \gamma_{l}, \quad l=1,2, \ldots, S, \\
\alpha_{n}>0, \quad n=1,2, \ldots, S, \quad \gamma_{1}=0, \quad \gamma_{l}>0, l=2,3, \ldots, S .
\end{gathered}
$$

If the $\alpha_{l}$ are ordered, the $\gamma_{l}$ obey $\alpha_{l}<\gamma_{l+1}<\alpha_{l+1}, \quad l=1,2 \ldots, S-1$ [14]. Then,

$$
\begin{aligned}
F_{p}\left(s_{1}, s_{2}\right) & =2 H_{\infty} \sum_{n, l=1}^{S} \frac{N_{p}\left(i \alpha_{n},-i \alpha_{l}\right)}{\alpha_{n}+\alpha_{l}} e^{-\alpha_{n} s_{1}-\alpha_{l} s_{2}}, \\
N_{p}\left(i \alpha_{n},-i \alpha_{l}\right) & =\frac{\alpha_{n} \alpha_{l} \prod_{j=2}^{S}\left\{\alpha_{n} \alpha_{l}+\gamma_{j}^{2}\right\}}{\prod_{\substack{j=1 \\
j \neq n}}^{S}\left(\alpha_{n}-\alpha_{j}\right) \prod_{\substack{j=1 \\
j \neq l}}^{S}\left(\alpha_{l}-\alpha_{j}\right)} .
\end{aligned}
$$

For one singularity at $i \alpha$ we obtain 14

$$
\psi_{p}(t)=\phi(t)+\frac{1}{2} H_{\infty} \alpha\left\{\int_{0}^{\infty} E_{r}^{t}(s) e^{-\alpha s} d s\right\}^{2}
$$

which is equal to the minimum free energy of Day [39] and the maximum free energy; there are no zeros in this case. Also, the rate of dissipation is given by

$$
D_{p}(t)=H_{\infty}\left\{\alpha \int_{0}^{\infty} E_{r}^{t}(s) e^{-\alpha s} d s\right\}^{2}
$$

Let us consider the case of two poles, but include not only the case where both poles and the single zero (other than the one at the origin) are on the positive imaginary axis, in other words formula (9.14) for $S=2$, but also where $\zeta_{1}=-\bar{\zeta}_{2}$ and $\eta_{2}=i \gamma$. Note that in the latter case the zero is also on the imaginary axis because if it is off the axis, it must have a partner. In both cases, the zero is denoted $i \gamma$. We let $N_{p}\left(\zeta_{n}, \bar{\zeta}_{l}\right)$ be the $n l$ component of the matrix $\mathbf{N}_{p}$ given by

$$
\mathbf{N}_{p}=\frac{1}{\left|\zeta_{1}-\zeta_{2}\right|^{2}}\left(\begin{array}{cc}
\left|\zeta_{1}\right|^{2}\left(\left|\zeta_{1}\right|^{2}+\gamma^{2}\right) & -\zeta_{1} \bar{\zeta}_{2}\left(\zeta_{1} \bar{\zeta}_{2}+\gamma^{2}\right) \\
-\zeta_{2} \bar{\zeta}_{1}\left(\zeta_{2} \bar{\zeta}_{1}+\gamma^{2}\right) & \left|\zeta_{2}\right|^{2}\left(\left|\zeta_{2}\right|^{2}+\gamma^{2}\right)
\end{array}\right)
$$

which is positive-definite. The matrix in $\left({ }^{9.13}\right)_{2}$ with $n l$ component $\frac{i N_{p}\left(\zeta_{n}, \bar{\zeta}_{l}\right)}{\zeta_{n}-\bar{\zeta}_{l}}$ is

$$
\mathbf{N}_{\psi}=\frac{i}{\left|\zeta_{1}-\zeta_{2}\right|^{2}}\left(\begin{array}{cc}
\frac{\left|\zeta_{1}\right|^{2}\left(\left|\zeta_{1}\right|^{2}+\gamma^{2}\right)}{2 i \operatorname{Im} \zeta_{1}} & -\frac{\zeta_{1} \bar{\zeta}_{2}\left(\zeta_{1} \bar{\zeta}_{2}+\gamma^{2}\right)}{\zeta_{1}-\bar{\zeta}_{2}} \\
-\frac{\zeta_{2} \bar{\zeta}_{1}\left(\zeta_{2} \bar{\zeta}_{1}+\gamma^{2}\right)}{\zeta_{2}-\bar{\zeta}_{1}} & \frac{\left|\zeta_{2}\right|^{2}\left(\left|\zeta_{2}\right|^{2}+\gamma^{2}\right)}{2 i I m \zeta_{2}}
\end{array}\right)
$$

More explicitly, for the two cases, we have

(a) $\zeta_{1}=i \alpha_{1}, \quad \zeta_{2}=i \alpha_{2}$,

$$
\begin{aligned}
& \mathbf{N}_{p}=\frac{1}{\left(\alpha_{1}-\alpha_{2}\right)^{2}}\left(\begin{array}{cc}
\alpha_{1}^{2}\left(\alpha_{1}^{2}+\gamma^{2}\right) & -\alpha_{1} \alpha_{2}\left(\alpha_{1} \alpha_{2}+\gamma^{2}\right) \\
-\alpha_{2} \alpha_{1}\left(\alpha_{2} \alpha_{1}+\gamma^{2}\right) & \alpha_{2}^{2}\left(\alpha_{2}^{2}+\gamma^{2}\right)
\end{array}\right) \\
& \mathbf{N}_{\psi}=\frac{1}{\left(\alpha_{1}-\alpha_{2}\right)^{2}}\left(\begin{array}{cc}
\frac{1}{2} \alpha_{1}\left(\alpha_{1}^{2}+\gamma^{2}\right) & -\frac{\alpha_{1} \alpha_{2}\left(\alpha_{1} \alpha_{2}+\gamma^{2}\right)}{\alpha_{1}+\alpha_{2}} \\
-\frac{\alpha_{1} \alpha_{2}\left(\alpha_{1} \alpha_{2}+\gamma^{2}\right)}{\alpha_{1}+\alpha_{2}} & \frac{1}{2} \alpha_{2}\left(\alpha_{2}^{2}+\gamma^{2}\right)
\end{array}\right),
\end{aligned}
$$


and

$$
\begin{aligned}
& \text { (b) } \zeta_{2}=-\bar{\zeta}_{1}, \\
& \mathbf{N}_{p}=\frac{1}{4\left(\operatorname{Re} \zeta_{1}\right)^{2}}\left(\begin{array}{cc}
\left|\zeta_{1}\right|^{2}\left(\left|\zeta_{1}\right|^{2}+\gamma^{2}\right) & \zeta_{1}^{2}\left(-\zeta_{1}^{2}+\gamma^{2}\right) \\
\bar{\zeta}_{1}^{2}\left(-\bar{\zeta}_{1}^{2}+\gamma^{2}\right) & \left|\zeta_{1}\right|^{2}\left(\left|\zeta_{1}\right|^{2}+\gamma^{2}\right)
\end{array}\right), \\
& \mathbf{N}_{\psi}=\frac{1}{8\left(\operatorname{Re} \zeta_{1}\right)^{2}}\left(\begin{array}{cc}
\frac{\left|\zeta_{1}\right|^{2}\left(\left|\zeta_{1}\right|^{2}+\gamma^{2}\right)}{I m \zeta_{1}} & i \zeta_{1}\left(-\zeta_{1}^{2}+\gamma^{2}\right) \\
-i \bar{\zeta}_{1}\left(-\bar{\zeta}_{1}^{2}+\gamma^{2}\right) & \frac{\left|\zeta_{1}\right|^{2}\left(\left|\zeta_{1}\right|^{2}+\gamma^{2}\right)}{I m \zeta_{1}}
\end{array}\right) .
\end{aligned}
$$

For cases (a) and (b), both matrices are positive-definite. Note that case (b) corresponds to a relaxation function derivative with one strictly decaying exponential multiplying a cosine function.

\section{REFERENCES}

[1] A.J. Staverman and F. Schwarzl, Thermodynamics of viscoelastic behaviour, Proc. Konink. Nederl. Akad. Wettensch. B55, 474-485 (1952).

[2] S. Breuer and E.T. Onat, On recoverable work in linear viscoelasticity, Z. Angew. Math. Phys. 15, 13-21 (1964). MR 0178644(31:2901)

[3] S. Breuer and E.T. Onat, On the determination of free energy in linear viscoelasticity, Z. Angew. Math. Phys. 15 184-191 (1964). MR0178645 (31:2902)

[4] B.D. Coleman and D.R. Owen, A Mathematical foundation for thermodynamics, Arch. Rational Mech. Anal. 54, 1-104 (1974). MR0395502 (52:16299)

[5] B.D. Coleman and D.R. Owen, On thermodynamics and elastic-plastic materials, Arch. Rational Mech. Anal. 59, 25-51 (1975). MR0381526 (52:2419)

[6] W. A. Day, An objection to using entropy as a primitive concept in continuum mechanics, Acta Mech. 27, 251-255 (1977).

[7] D. Graffi and M. Fabrizio, Non unicità dell'energia libera per i materiali viscoelastici, Atti Acc. Naz. Lincei 83, 209-214 (1990). MF,1142460 (93a:73037)

[8] A. Morro and M. Vianello, Minimal and maximal free energy for materials with memory, Boll. Un. Mat. Ital. 4A, 45-55 (1990). MR1047512 (91b:73012)

[9] M. Fabrizio and A. Morro, Mathematical Problems in Linear Viscoelasticity, SIAM, Philadelphia, 1992. MR:1153021 (93a:73034)

[10] M. Fabrizio, C. Giorgi and A. Morro, Free energies and dissipation properties for systems with memory, Arch. Rational Mech. Anal. 125, 341-373 (1994). MR.1253168 (95j:73012)

[11] G. Del Piero and L. Deseri, On the analytic expression of the free energy in linear viscoelasticity, J. Elasticity 43, 247-278 (1996). MR1415545 (97g:73042)

[12] G. Del Piero and L. Deseri, On the concepts of state and free energy in linear viscoelasticity, Arch. Rational Mech. Anal. 138, 1-35 (1997). MR.1463802 (98i:73023)

[13] L. Deseri, G. Gentili, J. M. Golden, An explicit formula for the minimum free energy in linear viscoelasicity, J. Elasticity 54, 141-185 (1999). MR1728444 (2001i:74012)

[14] J. M. Golden, Free energies in the frequency domain: the scalar case, Quart. Appl. Math. 58, 127-150 (2000). MR1739041 (2001e:74025)

[15] M. Fabrizio and J.M. Golden, Maximum and minimum free energies and the concept of a minimal state, Rendiconti di Matematica 20, 131-165 (2000). MR1823095 (2002a:74029)

[16] J.M. Golden, Consequences of non-uniqueness in the free energy of materials with memory, Int. J. Engng Sci. 39, 53-70 (2001). MR.1801797(2001i:74014)

[17] M. Fabrizio and J.M. Golden, Maximum and minimum free energies for a linear viscoelastic material, Quart. Appl. Math. 60, 341 - 381 (2002). MR1900497 (2003b:74013)

[18] M. Fabrizio, G. Gentili and J.M. Golden, The minimum free energy for a class of compressible viscoelastic fluids, Differential and Integral Equations, 7, 319-342 (2002). MR.1867690|(2002k:76013)

[19] M. Fabrizio and J.M. Golden, Minimum Free Energies for Materials with Finite Memory, J. Elasticity 72, 121-143 (2003). MR2064221 
[20] M. Fabrizio, G. Gentili and J.M. Golden, Free energies for linear non-isothermal materials with memory, Math. Comput. Modelling 39, 219-253 (2004). MR2037395 (2004k:74004)

[21] L. Deseri and J.M. Golden, The minimum free energy for continuous spectrum materials, in preparation.

[22] W. Noll, A new mathematical theory of simple materials, Arch. Rational Mech. Anal. 48, 1-50 (1972). MR0445985 (56:4318)

[23] C. Banfi, Su una nuova impostazione per l'analisi dei sistemi ereditari, Ann. Univ. Ferrara, N. Ser., Sez. VII 23, 29-38 (1977).

[24] D. Graffi and M. Fabrizio, Sulla nozione di stato materiali viscoelastici di tipo 'rate', Atti Accad. Naz. Lincei 83, 201-208 (1990). MF1142459 (93a:73036)

[25] J. D. Ferry, Viscoelastic Properties of Polymers, John Wiley, New York, 3rd edition, 1980.

[26] R.M. Christensen, Theory of Viscoelasticity: An Introduction, 2nd ed., Academic Press, New York, 1982.

[27] D. Bland, Theory of Linear Viscoelasticity, Pergamon, London, 1960. MR.0110314 (22:1194)

[28] S.C. Hunter, Tentative equations for the propagation of stress, strain and temperature fields in viscoelastic solids, J. Mech. Phys. Solids, 9, 39-51 (1961). MR0165780 (29:3060)

[29] J.M. Golden and G.A.C. Graham, Boundary Value Problems in Linear Viscoelasticity, SpringerVerlag, Berlin, 1988. MR0958684 (89i:73035)

[30] L. Deseri, G. Gentili, J. M. Golden, Free energies and Saint-Venant's principle in linear viscoelasticity, submitted for publication.

[31] G. Gentili, Maximum recoverable work, minimum free energy and state space in linear viscoelasticity, Quart. Appl. Math. 60, 153-182 (2002). MR1878264 (2002m:74011)

[32] M. E. Gurtin and I. Herrera, On dissipation inequalities and linear viscoelasticity, Quart. Appl. Math. 23, 235-245 (1988). MR0189346 (32:6772)

[33] W.A. Day, Thermodynamics based on a work axiom, Arch. Rational Mech. Anal. 31, 1-34 (1968).

[34] B.D. Coleman, Thermodynamics of materials with memory, Arch. Rational Mech. Anal. 17, 1-45 (1964). MR0171419 (30:1650)

[35] D. Graffi, Sull'expressione analitica di alcune grandezze termodinamiche nei materiali con memoria, Rend. Sem. Mat. Univ. Padova 68, 17-29 (1982). MR.0702143 (84i:73093)

[36] N. I. Muskhelishvili, Singular Integral Equations, Noordhoff, Groningen, 1953. MR0058845 $(15: 434 \mathrm{e})$

[37] M. Fabrizio, C. Giorgi and A. Morro, Internal dissipation, relaxation property and free energy in materials with fading memory. J. Elasticity 40, 107-122 (1995). MR1364749 (96g:73008)

[38] E.T. Whittaker and G.N. Watson, A Course of Modern Analysis, Cambridge University Press, 1963 MR.0178117 (31:2375)

[39] W.A. Day, The thermodynamics of materials with memory, Materials with Memory, D. Graffi ed., Liguori, Napoli, 1979. 